\title{
Pricing and Hedging Quanto Options in Energy Markets
}

\author{
Benth, Fred Espen; Lange, Nina; Myklebust, Tor Åge
}

Document Version

Final published version

Published in:

Journal of Energy Markets

Publication date:

2015

License

Unspecified

Citation for published version (APA):

Benth, F. E., Lange, N., \& Myklebust, T. Å. (2015). Pricing and Hedging Quanto Options in Energy Markets. Journal of Energy Markets, 8(1), 1-35.

Link to publication in CBS Research Portal

\section{General rights}

Copyright and moral rights for the publications made accessible in the public portal are retained by the authors and/or other copyright owners and it is a condition of accessing publications that users recognise and abide by the legal requirements associated with these rights.

Take down policy

If you believe that this document breaches copyright please contact us (research.lib@cbs.dk) providing details, and we will remove access to the work immediately and investigate your claim. 
inst

journals

\title{
Research Paper
}

\section{Pricing and hedging quanto options in energy markets}

\author{
Fred Espen Benth ${ }^{1}$, Nina Lange ${ }^{2}$ and Tor Åge Myklebust ${ }^{3}$ \\ ${ }^{1}$ Department of Mathematics, University of Oslo, PO Box 1053, Blindern, \\ 0316 Oslo, Norway; email: fredb @ math.uio.no \\ ${ }^{2}$ Department of Finance, Copenhagen Business School, Solbjerg Plads 3, \\ 2000 Frederiksberg, Denmark; email: nl.fi@ cbs.dk \\ ${ }^{3}$ Institute of Population-Based Cancer Research, Cancer Registry of Norway, PO Box 5313, \\ Majorstuen, 0369 Oslo, Norway; email: tor.age.myklebust@ kreftregisteret.no
}

(Received January 31, 2014; revised April 11, 2014; accepted July 11, 2014)

\begin{abstract}
In energy markets, the use of quanto options has increased significantly in recent years. The payoff from such options are typically written on an underlying energy index and a measure of temperature. They are suited to managing the joint price and volume risk in energy markets. Using a Heath-Jarrow-Morton approach, we derive a closed-form option pricing formula for energy quanto options under the assumption that the underlying assets are lognormally distributed. Our approach encompasses several interesting cases, such as geometric Brownian motions and multifactor spot models. We also derive Delta and Gamma expressions for hedging. Further, we illustrate the use of our model by an empirical pricing exercise using New York Mercantile Exchange-traded natural gas futures and Chicago Mercantile Exchange-traded heating degree days futures for New York.
\end{abstract}

Keywords: gas derivatives; temperature derivatives; energy quanto options; quantity risk; pricing; hedging. 


\section{INTRODUCTION}

Many industries are exposed to the variability of the weather. Take, as an example, a gas distribution company that operates in an open wholesale market. Their planned sales volumes per day and the market price are the two main factors to which they are exposed. If, for example, one of the winter months turns out to be warmer than usual, the demand for gas would drop. This decline in demand would probably also affect the market price for gas, leading to a drop in gas price. The firm would make a loss compared with their planned revenue, which is equal to the shortfall in demand multiplied by the difference between the retail price at which they would have sold had their customers bought the gas, and the market price at which they must now sell their excess gas. So, they face not only a direct weather effect, eg, the lower demand, but also an indirect effect through the drop in market prices. The above example clearly illustrates that the adverse movements in market price and demand due to higher temperatures represent a kind of correlation risk, which is difficult to properly hedge against, as it leads to a heavier tailed profit-and-loss distribution. Using standard weather derivatives as offered by the Chicago Mercantile Exchange (CME) would most likely represent an imperfect and rather expensive hedging strategy, as it accounts only for the direct earnings effect from the change in demand, and not the indirect earnings effect from price changes. If standardized weather products are insufficient as hedging tools, the companies must turn to over-the-counter (OTC) markets for weather derivatives. Davis (2010) and Pérez-González and Yun (2013) refer to surveys conducted by the Weather Risk Management Association (WRMA) and reports from the CME about market sizes and expected development: ${ }^{1}$ the market for standardized weather derivatives peaked in 2007 with a total volume of trades close to 930000 and a corresponding notional value of US $\$ 17.9$ billion. Since 2008, the market for standardized contracts has experienced severe retrenchment. In 2009, the total volume of trades dipped below 500000 , amounting to a notional value of around US\$5.3 billion. A big part of this sharp decline is attributed to the substantial increase, eg, 30\% from 2010 to 2011, in the market for tailor-made contracts, especially the quantity-adjusting weather contracts ("quantos"). Contracts of this type worth US\$100 million have been reported. Market participants indicate that the demand for quanto contracts is international, with transactions being executed in the United States, Europe, Australia and South America. In 2010, the WRMA believed that the developing market in India alone had a potential value of US $\$ 2.35$ billion.

\footnotetext{
${ }^{1}$ Although the reported numbers are small compared with other markets, weather-exposed utilities can use weather derivatives to reduce extreme losses from weather incidents and increase the valuation of the company (see Pérez-González and Yun (2013) for an extensive study of the effect of weather derivatives on firm value, investments and leverage).
} 
The label "quanto options" has traditionally been assigned to a class of derivatives in financial markets where the investor wishes to be exposed to price movements in the foreign asset without the corresponding exchange rate risk. The pricing of currency quanto options has been extensively researched and dates back to the original work of Garman and Kohlhagen (1983). Although the same term is used for the specific type of energy options that we study in this paper, these two types of derivatives contracts are different: a typical currency quanto option has a regular call-put payoff structure, whereas the energy quanto options we study have a payoff structure similar to a product of call-put options, and energy quanto options are therefore mainly used to hedge exposure to the joint price and volume risk. ${ }^{2}$ In comparison with studies of currency quantos, research related to the pricing of quanto options in energy markets is scarce. One exception is Caporin et al (2012), who propose a bivariate time-series model to capture the joint dynamics of energy prices and temperature. In particular, they model the energy price and the average temperature using a sophisticated parameter-intensive econometric model. Since they aim to capture features such as seasonality in means and variances, long memory, autoregressive patterns and dynamic correlations, the complexity of their model leaves no other option than simulation-based procedures to calculate prices. Moreover, they leave the issue of how we should hedge such options unanswered.

In order for quanto contracts to provide a superior risk management tool compared with standardized futures contracts, it is crucial that there is a significant correlation between the two underlying assets. In energy markets, the payoff of a quanto option is determined by the level of both the energy price and an index related to weather. This correlation has been studied by, for example, Engle et al (1992), who documents that temperature is important in forecasting electricity prices, and Timmer and Lamb (2007), who document a strong relationship between natural gas prices and heating degree days (HDD).

In this paper, we also study the pricing of energy quanto options. However, unlike Caporin et al (2012), we derive analytical solutions to the option pricing problem. Such closed-form solutions are easy to implement, fast to calculate and, most importantly, they give a clear answer as to how the energy quanto option should be properly hedged. We convert the pricing problem by using traded futures contracts on energy and a temperature index as underlying assets, rather than energy spot prices and temperature. We are able to do this because the typical energy quanto options have a payoff that can be represented as an "Asian" structure on the energy spot price and the temperature index. The markets for energy and weather organize futures with delivery periods, which will coincide with the aggregate or average spot price and

2 This double-call structure was also studied by Jørgensen (2007) for the case of interest rates and stock prices. 
temperature index at the end of the delivery period. Hence, any "Asian payoff" on the spot and temperature for a quanto option can be viewed as a "European payoff" on the corresponding futures contracts. This insight is the key to our solution and the main contribution of this paper. The analytical solution also gives the desirable feature that we can hedge the quanto option in terms of traded instruments, namely the underlying futures contracts that - unlike temperature and spot power/gas - can be easily bought and held.

Using a Heath-Jarrow-Morton (HJM) approach, we derive options prices under the assumption that futures dynamics are lognormally distributed with a possibly timevarying volatility. Furthermore, we explicitly derive Delta-hedging and cross-Gamma hedging parameters. Our approach encompasses several models for the underlying futures prices, such as the standard bivariate geometric Brownian motion (GBM) and the two-factor model proposed in papers such as Schwartz and Smith (2000), Sørensen (2002) and Lucia and Schwartz (2002). The latter class of models allows for time-varying volatility, which is a stylized fact for many commodities. We include an extensive empirical example to illustrate our findings. Using futures contracts on natural gas and the HDD temperature index, we estimate relevant parameters in the seasonal two-factor model of Sørensen (2002) based on data collected from the New York Mercantile Exchange (NYMEX) and the CME. We compute prices for various energy quanto options and benchmark these against products of plain-vanilla European options on gas and HDD futures. The latter can be priced by the classical Black (1976) option pricing formula and corresponds to the case of the energy quanto option for independent gas and temperature futures. In Section 2, we discuss the structure of energy quanto options and introduce the pricing problem. In Section 3, we derive the pricing and hedging formulas and show how the model of futures price dynamics in Sørensen (2002) is a special case of our general framework. Section 4 presents the empirical case study, and Section 5 concludes.

\section{THE CONTRACT STRUCTURE AND PRICING OF ENERGY QUANTO OPTIONS}

In this section, we first discuss typical examples of energy quanto options. We then argue that the pricing problem can be simplified using standardized futures contracts as underlying assets.

\subsection{Contract structure}

Most energy quanto contracts have payoffs that are triggered by two underlying "assets", temperature and energy price. Since these contracts are tailor made, rather than standardized, the contract design varies. In its simplest form, a quanto contract 
TABLE 1 A specification of a typical energy quanto option.

\begin{tabular}{lllllll}
\hline & Nov & Dec & Jan & Feb & Mar \\
\hline (a) High strike (HDD) & $\bar{K}_{I}^{11}$ & $\bar{K}_{I}^{12}$ & $\bar{K}_{I}^{1}$ & $\bar{K}_{I}^{2}$ & $\bar{K}_{I}^{3}$ \\
(b) Low strike (HDD) & $\underline{K}_{I}^{11}$ & $\underline{K}_{I}^{12}$ & $\underline{K}_{I}^{1}$ & $\underline{K}_{I}^{2}$ & $\underline{K}_{I}^{3}$ \\
(a) High strike (price/mmBtu) & $\bar{K}_{E}^{11}$ & $\bar{K}_{E}^{12}$ & $\bar{K}_{E}^{1}$ & $\bar{K}_{E}^{2}$ & $\bar{K}_{E}^{3}$ \\
(b) Low strike (price/mmBtu) & $\underline{K}_{E}^{11}$ & $\underline{K}_{E}^{12}$ & $\underline{K}_{E}^{1}$ & $\underline{K}_{E}^{2}$ & $\underline{K}_{E}^{3}$ \\
Volume (mmBtu) & 200 & 300 & 500 & 400 & 250 \\
\hline
\end{tabular}

The underlying process triggering payoffs to the option holder is the accumulated number of HDD $I$ and the monthly index gas price $E$. As an example, the payoff for November will be (a) in cold periods, $\max \left(I-\bar{K}_{I}, 0\right) \times \max (E-$ $\left.\bar{K}_{E}, 0\right) \times$ volume, and (b) in warm periods, $\max \left(\underline{K}_{I}-I, 0\right) \times \max \left(\underline{K}_{E}-E, 0\right) \times$ volume. We see that the option pays out if both the underlying temperature and price variables exceed (dip below) the high strikes (low strikes).

has a payoff function $S$ :

$$
S=\left(T_{\mathrm{var}}-T_{\mathrm{fix}}\right) \times\left(E_{\mathrm{var}}-E_{\mathrm{fix}}\right) .
$$

Payoff is determined by the difference between some variable temperature measure $\left(T_{\text {var }}\right)$ and some fixed temperature measure $\left(T_{\text {fix }}\right)$ multiplied by the difference between variable and fixed energy price ( $E_{\mathrm{var}}$ and $\left.E_{\mathrm{fix}}\right)$. Note that the payoff might be negative, indicating that the buyer of the contract pays the required amount to the seller.

Entering into a quanto contract of this type might be risky, since the downside may potentially become large. For hedging purposes, it seems more reasonable to buy a quanto structure with optionality, thereby eliminating all downside risk. In Table 1, we show a typical example of how a quanto option might be structured (see also Caporin et al (2012) for a discussion of the design of the energy quanto option). The example contract has a payoff that is triggered by an average gas price denoted $E$ (defined as the average of daily prices for the last month). It also offers an exposure to temperature through the accumulated number of HDD in the corresponding month. The HDD index is commonly used as the underlying variable for temperature derivatives and is defined as how much the average temperature over a day has dropped below a preset level. We denote the accumulated number of HDD over interval $\left[\tau_{1}, \tau_{2}\right]$ by $I_{\left[\tau_{1}, \tau_{2}\right]}$ :

$$
I_{\left[\tau_{1}, \tau_{2}\right]}=\sum_{t=\tau_{1}}^{\tau_{2}} \mathrm{HDD}_{t}=\sum_{t=\tau_{1}}^{\tau_{2}} \max \left(c-T_{t}, 0\right),
$$

where $c$ is some prespecified temperature threshold $\left(65^{\circ} \mathrm{F}\right.$ or $\left.18{ }^{\circ} \mathrm{C}\right)$ and $T_{t}$ is the mean temperature on day $t$. If the number of HDD $I$ and the average gas price $E$ are above the high strikes ( $\bar{K}_{I}$ and $\bar{K}_{E}$, respectively), the owner of the option would receive a payment equal to the prespecified volume multiplied by the actual number of HDD minus the strike $\bar{K}_{I}$ multiplied by the difference between the average energy 
price minus the strike price $\bar{K}_{E}$ if $E>\bar{K}_{E}$. On the other hand, if it is warmer than usual and the number of HDD dips below the lower strike of $\underline{K}_{I}$, and the energy price at the same time is lower than $\underline{K}_{E}$, the owner receives a payout equal to the volume multiplied by $\underline{K}_{I}$ minus the actual number of HDD multiplied by the difference between the strike price $\underline{K}_{E}$ and the average energy price. Note that the volume adjustment varies between months, reflecting the fact that "unusual" temperature changes might have a stronger impact on the option holder's revenue in the coldest months, such as December and January. Also note that the price strikes may vary between months.

This example illustrates why quanto options might be a good alternative to more standardized derivatives. The structure of the contracts takes into account the fact that extreme temperature variations might affect both demand and prices, and it compensates the owner of the option by making payoffs contingent on both prices and temperatures. The great possibility of tailoring these contracts provides potential customers with a powerful and efficient hedging instrument.

\subsection{Pricing using terminal value of futures}

As described above, energy quanto options have a payoff that is a function of two underlying assets, temperature and price. We focus on a class of energy quanto options that has a payoff function $f(E, I)$, where $E$ is an index of the energy price and $I$ is an index of temperature. To be more specific, we assume that the energy index $E$ is given as the average spot price over some measurement period $\left[\tau_{1}, \tau_{2}\right], \tau_{1}<\tau_{2}$,

$$
E=\frac{1}{\tau_{2}-\tau_{1}} \sum_{u=\tau_{1}}^{\tau_{2}} S_{u},
$$

where $S_{u}$ denotes the energy spot price. Further, we assume that the temperature index is defined as

$$
I=\sum_{u=\tau_{1}}^{\tau_{2}} g\left(T_{u}\right)
$$

for $T_{u}$ the temperature at time $u$ and $g$ some function. For example, if we want to consider a quanto option involving the HDD index, we choose $g(x)=\max (x-18,0)$. The quanto option is exercised at time $\tau_{2}$, and its arbitrage-free price $C_{t}$ at time $t \leqslant \tau_{2}$ is defined by the following expression:

$$
C_{t}=\mathrm{e}^{-r\left(\tau_{2}-t\right)} \mathbb{E}_{t}^{\mathbb{Q}}\left[f\left(\frac{1}{\tau_{2}-\tau_{1}} \sum_{u=\tau_{1}}^{\tau_{2}} S_{u}, \sum_{u=\tau_{1}}^{\tau_{2}} g\left(T_{u}\right)\right)\right] .
$$

Here, $r>0$ denotes the risk-free interest rate, which, for simplicity, we assume is constant. The pricing measure is denoted $\mathbb{Q}$, and $\mathbb{E}_{t}^{\mathbb{Q}}[\cdot]$ is the expectation operator with respect to $\mathbb{Q}$, conditioned on the market information at time $t$ given by the filtration $\mathcal{F}_{t}$. 
We now argue how to relate the price of the quanto option to futures contracts on the energy and temperature indexes $E$ and $I$. Observe that the price at time $t \leqslant \tau_{2}$ of a futures contract written on some energy price (eg, natural gas) with delivery period $\left[\tau_{1}, \tau_{2}\right]$ is given by

$$
F_{t}^{E}\left(\tau_{1}, \tau_{2}\right)=\mathbb{E}_{t}^{\mathbb{Q}}\left[\frac{1}{\tau_{2}-\tau_{1}} \sum_{u=\tau_{1}}^{\tau_{2}} S_{u}\right] .
$$

At time $t=\tau_{2}$, we find from the conditional expectation that

$$
F_{\tau_{2}}^{E}\left(\tau_{1}, \tau_{2}\right)=\frac{1}{\tau_{2}-\tau_{1}} \sum_{u=\tau_{1}}^{\tau_{2}} S_{u},
$$

ie, the futures price is exactly equal to what is being delivered. Applying the same argument to a futures contract written on the temperature index, with price dynamics denoted $F_{t}^{I}\left(\tau_{1}, \tau_{2}\right)$, we immediately see that the following must be true for the quanto option price:

$$
\begin{aligned}
C_{t} & =\mathrm{e}^{-r\left(\tau_{2}-t\right)} \mathbb{E}_{t}^{\mathbb{Q}}\left[f\left(\frac{1}{\tau_{2}-\tau_{1}} \sum_{u=\tau_{1}}^{\tau_{2}} S_{u}, \sum_{u=\tau_{1}}^{\tau_{2}} g\left(T_{u}\right)\right)\right] \\
& =\mathrm{e}^{-r\left(\tau_{2}-t\right)} \mathbb{E}_{t}^{\mathbb{Q}}\left[f\left(F_{\tau_{2}}^{E}\left(\tau_{1}, \tau_{2}\right), F_{\tau_{2}}^{I}\left(\tau_{1}, \tau_{2}\right)\right)\right] .
\end{aligned}
$$

Equation (2.4) shows that the price of a quanto option with payoff being a function of the energy index $E$ and temperature index $I$ must be the same as if the payoff was a function of the terminal values of two futures contracts written on the energy and temperature indexes, and with the delivery period being equal to the contract period specified by the quanto option. Hence, we view the quanto option as an option written on the two futures contracts, rather than on the two indexes. This is advantageous from the point of view that the futures are traded financial assets. We note in passing that we may extend the above argument to quanto options where the measurement periods of the energy and temperature indexes are not the same.

To compute the price in (2.4), we must have a model for the futures price dynamics $F_{t}^{E}\left(\tau_{1}, \tau_{2}\right)$ and $F_{t}^{I}\left(\tau_{1}, \tau_{2}\right)$. The dynamics must account for the dependency between the two futures, as well as their marginal behavior. The pricing of the energy quanto option has thus been transferred from modeling the joint spot energy and temperature dynamics, followed by computing the $\mathbb{Q}$ expectation of an index of these, to modeling the joint futures dynamics and pricing a European-type option on these. The former approach is similar to pricing an Asian option, which for most relevant models and cases is a highly difficult task. We remark also that, by modeling and estimating the futures dynamics to market data, we can easily obtain the market-implied pricing 
measure $\mathbb{Q}$. We will see this in practice in Section 4 , where we analyze the case of gas and HDD futures. If we choose to model the underlying energy spot prices and temperature dynamics, we obtain a dynamics under the market probability $\mathbb{P}$, rather than under the pricing measure $\mathbb{Q}$. Additional hypotheses must be made in the model to obtain this. Moreover, for most interesting cases, the quanto option must be priced by Monte Carlo or some other computationally demanding method (see Caporin et al 2012). Finally, but no less importantly, with the representation in (2.4) at hand we can discuss the issue of hedging energy quanto options in terms of the underlying futures contracts.

In many energy markets, futures contracts are not traded within their delivery period. That means that we can only use the market for futures up to time $\tau_{1}$. This has a clear consequence for the possibility of hedging these contracts, as a hedging strategy will inevitably be a continuously rebalanced portfolio of the futures up to the exercise time $\tau_{2}$. As this is possible to perform only up to time $\tau_{1}$ in many markets, we face an incomplete market situation where the quanto option cannot be hedged perfectly. Moreover, it is to be expected that the dynamics of the futures price has different characteristics within the delivery period than prior to the start of delivery, if it can be traded for times $t \in\left(\tau_{1}, \tau_{2}\right]$. The reason for this is that we have less uncertainty as the remaining delivery period of the futures decreases. In this paper, we will restrict our attention to the pricing of quanto options at times $t \leqslant \tau_{1}$. The entry time of such a contract most naturally takes place prior to the delivery period. However, for marking-to-market purposes, we are also interested in the price $C_{t}$ for $t \in\left(\tau_{1}, \tau_{2}\right]$. The issuer of the quanto option may be interested in hedging the exposure and may therefore also be concerned with the behavior of prices within the delivery period.

Before we start looking into the details of pricing quanto options, we will investigate an options contract of the type described in Section 2.1 in more detail. This contract covers a period of five months, from November through to March. Since this contract is essentially a sum of one-period contracts, we focus our attention on an option covering only one month in the delivery period $\left[\tau_{1}, \tau_{2}\right]$. Recall that the payoff in the contract is a function of some average energy price and accumulated number of HDD. From the discussion in the previous section, we know that, rather than using the spot price and HDD as underlying assets, we can instead use the terminal value of futures contracts written on price and HDD, respectively. The payoff function

$$
p\left(F_{\tau_{2}}^{E}\left(\tau_{1}, \tau_{2}\right), F_{\tau_{2}}^{I}\left(\tau_{1}, \tau_{2}\right), \bar{K}_{E}, \bar{K}_{I}, \underline{K}_{E}, \underline{K}_{I}\right)=p
$$

of this quanto contract is defined as

$$
\begin{aligned}
p=\gamma\left[\max \left(F_{\tau_{2}}^{E}\left(\tau_{1}, \tau_{2}\right)-\bar{K}_{E}, 0\right) \max \left(F_{\tau_{2}}^{I}\left(\tau_{1}, \tau_{2}\right)-\bar{K}_{I}, 0\right)\right. \\
\left.\quad+\max \left(\underline{K}_{E}-F_{\tau_{2}}^{E}\left(\tau_{1}, \tau_{2}\right), 0\right) \max \left(\underline{K}_{I}-F_{\tau_{2}}^{I}\left(\tau_{1}, \tau_{2}\right), 0\right)\right],
\end{aligned}
$$


where $\gamma$ is the contractual volume adjustment factor. Note that the payoff function in this contract consists of two parts, the first taking care of the situation in which temperatures are colder (and prices higher) than usual, and the second taking care of the situation in which temperatures are warmer (and prices lower) than usual. The first part is the product of two call options, whereas the second part is the product of two put options. To illustrate our pricing approach in the simplest way possible, it suffices to look at the product call structure with the volume adjuster $\gamma$ normalized to 1 , ie, we want to price an option with the following payoff function:

$$
\begin{aligned}
\hat{p}\left(F_{\tau_{2}}^{E}\left(\tau_{1}, \tau_{2}\right), F_{\tau_{2}}^{I}\left(\tau_{1}, \tau_{2}\right), \bar{K}_{E}, \bar{K}_{I}\right) & \\
& =\max \left(F_{\tau_{2}}^{E}\left(\tau_{1}, \tau_{2}\right)-\bar{K}_{E}, 0\right) \max \left(F_{\tau_{2}}^{I}\left(\tau_{1}, \tau_{2}\right)-\bar{K}_{I}, 0\right) .
\end{aligned}
$$

In the remainder of this paper, we will focus on this particular choice of payoff function for the energy quanto option. It corresponds to choosing the function $f$ as $f(E, I)=\max \left(E-\bar{K}_{E}, 0\right) \max \left(I-\bar{K}_{I}, 0\right)$ in (2.4). Other combinations of putcall mixes, as well as different delivery periods for the energy and temperature futures, can easily be studied by a simple modification of what follows.

\section{ASSET PRICE DYNAMICS AND OPTION PRICES}

Suppose that the two futures price dynamics under the pricing measure $\mathbb{Q}$ can be expressed as

$$
\begin{gathered}
F_{T}^{E}\left(\tau_{1}, \tau_{2}\right)=F_{t}^{E}\left(\tau_{1}, \tau_{2}\right) \exp \left(\mu_{E}+X\right), \\
F_{T}^{I}\left(\tau_{1}, \tau_{2}\right)=F_{t}^{I}\left(\tau_{1}, \tau_{2}\right) \exp \left(\mu_{I}+Y\right),
\end{gathered}
$$

where $t \leqslant T \leqslant \tau_{2}$, and $X, Y$ are two random variables independent of $\mathcal{F}_{t}$ but dependent on $t, T, \tau_{1}$ and $\tau_{2}$. We suppose that $(X, Y)$ is a bivariate normally distributed random variable with mean zero and covariance structure dependent on $t, T$ and $\tau_{2}$. We define

$$
\sigma_{X}^{2}=\operatorname{var}(X), \quad \sigma_{Y}^{2}=\operatorname{var}(Y) \quad \text { and } \quad \rho_{X, Y}=\operatorname{corr}(X, Y) .
$$

Obviously, $\sigma_{X}, \sigma_{Y}$ and $\rho_{X, Y}$ are dependent on $t, T, \tau_{1}$ and $\tau_{2}$. Moreover, as the futures price is naturally a martingale under the pricing measure $\mathbb{Q}$, we have $\mu_{E}=-\sigma_{X}^{2} / 2$ and $\mu_{I}=-\sigma_{Y}^{2} / 2$.

Our general representation of the futures price dynamics (3.1) and (3.2) encompasses many interesting models. For example, a bivariate GBM looks like

$$
\begin{gathered}
F_{T}^{E}\left(\tau_{1}, \tau_{2}\right)=F_{t}^{E}\left(\tau_{1}, \tau_{2}\right) \exp \left(-\frac{1}{2} \sigma_{E}^{2}(T-t)+\sigma_{E}\left(W_{T}-W_{t}\right)\right), \\
F_{T}^{I}\left(\tau_{1}, \tau_{2}\right)=F_{t}^{I}\left(\tau_{1}, \tau_{2}\right) \exp \left(-\frac{1}{2} \sigma_{I}^{2}(T-t)+\sigma_{I}\left(B_{T}-B_{t}\right)\right),
\end{gathered}
$$


with two Brownian motions $W$ and $B$ being correlated. We can easily associate this GBM to the general setup above by setting

$$
\begin{aligned}
\mu_{E} & =-\frac{1}{2} \sigma_{E}^{2}(T-t), & \mu_{I} & =-\frac{\sigma_{I}^{2}(T-t)}{2}, \\
\sigma_{X} & =\sigma_{E} \sqrt{T-t}, & \sigma_{Y} & =\sigma_{I} \sqrt{T-t}
\end{aligned}
$$

with $\rho_{X, Y}$ being the correlation between the two Brownian motions. In Section 3.2, we show that the two-factor model by Schwartz and Smith (2000) and the extension by Sørensen (2002) also fit this framework.

\subsection{A general solution for the quanto option price and hedge}

The price of the quanto option at time $t$ is

$$
C_{t}=\mathrm{e}^{-r\left(\tau_{2}-t\right)} \mathbb{E}_{t}^{\mathbb{Q}}\left[\hat{p}\left(F_{\tau_{2}}^{E}\left(\tau_{1}, \tau_{2}\right), F_{\tau_{2}}^{I}\left(\tau_{1}, \tau_{2}\right), \bar{K}_{E}, \bar{K}_{I}\right)\right],
$$

where the notation $\mathbb{E}^{\mathbb{Q}}$ denotes that the expectation is taken under the pricing measure $\mathbb{Q}$. Given these assumptions, Proposition 3.1 states the closed-form solution of the energy quanto option.

Proposition 3.1 For two assets following the dynamics given by (3.1) and (3.2), the time $t$ market price of a European energy quanto option with exercise at time $\tau_{2}$ and payoff described by (2.6) is given by

$$
\begin{aligned}
C_{t}=\mathrm{e}^{-r\left(\tau_{2}-t\right)} & \left(F_{t}^{E}\left(\tau_{1}, \tau_{2}\right) F_{t}^{I}\left(\tau_{1}, \tau_{2}\right) \mathrm{e}^{\rho_{X, Y} \sigma_{X} \sigma_{Y}} M\left(y_{1}^{* * *}, y_{2}^{* * *} ; \rho_{X, Y}\right)\right. \\
& -F_{t}^{E}\left(\tau_{1}, \tau_{2}\right) \bar{K}_{I} M\left(y_{1}^{* *}, y_{2}^{* *} ; \rho_{X, Y}\right) \\
& \left.-F_{t}^{I}\left(\tau_{1}, \tau_{2}\right) \bar{K}_{E} M\left(y_{1}^{*}, y_{2}^{*} ; \rho_{X, Y}\right)+\bar{K}_{E} \bar{K}_{I} M\left(y_{1}, y_{2} ; \rho_{X, Y}\right)\right),
\end{aligned}
$$

where

$$
\begin{gathered}
y_{1}=\frac{\log \left(F_{t}^{E}\left(\tau_{1}, \tau_{2}\right)\right)-\log \left(\bar{K}_{E}\right)-\sigma_{X}^{2} / 2}{\sigma_{X}}, \\
y_{2}=\frac{\log \left(F_{t}^{I}\left(\tau_{1}, \tau_{2}\right)\right)-\log \left(\bar{K}_{I}\right)-\sigma_{Y}^{2} / 2}{\sigma_{Y}}, \\
y_{1}^{*}=y_{1}+\rho_{X, Y} \sigma_{Y}, \quad y_{2}^{*}=y_{2}+\sigma_{Y}, \\
y_{1}^{* *}=y_{1}+\sigma_{X}, \quad y_{2}^{* *}=y_{2}+\rho_{X, Y} \sigma_{X}, \\
y_{1}^{* * *}=y_{1}+\rho_{X, Y} \sigma_{Y}+\sigma_{X}, \quad y_{2}^{* * *}=y_{2}+\rho_{X, Y} \sigma_{X}+\sigma_{Y} .
\end{gathered}
$$

Here, $M(x, y ; \rho)$ denotes the standard bivariate normal cumulative distribution function with correlation $\rho$. 
Proof Observe that the payoff function in (2.6) can be rewritten in the following way:

$$
\begin{aligned}
\hat{p}\left(F^{E}, F^{I},\right. & \left.\bar{K}_{E}, \bar{K}_{I}\right) \\
= & \max \left(F^{E}-\bar{K}_{E}, 0\right) \max \left(F^{I}-\bar{K}_{I}, 0\right) \\
= & \left(F^{E}-\bar{K}_{E}\right)\left(F^{I}-\bar{K}_{I}\right) 1_{\left\{F^{E}>\bar{K}_{E}\right\}} 1_{\left\{F^{I}>\bar{K}_{I}\right\}} \\
= & F^{E} F^{I} 1_{\left\{F^{E}>\bar{K}_{E}\right\}} 1_{\left\{F^{I}>\bar{K}_{I}\right\}}-F^{E} \bar{K}_{I} 1_{\left\{F^{E}>\bar{K}_{E}\right\}} 1_{\left\{F^{I}>\bar{K}_{I}\right\}} \\
& \quad-F^{I} \bar{K}_{E} 1_{\left\{F^{E}>\bar{K}_{E}\right\}} 1_{\left\{F^{I}>\bar{K}_{I}\right\}}+\bar{K}_{E} \bar{K}_{I} 1_{\left\{F^{E}>\bar{K}_{E}\right\}} 1_{\left\{F^{I}>\bar{K}_{I}\right\}} .
\end{aligned}
$$

The problem of finding the market price of the European quanto option is thus equivalent to the problem of calculating the expectations under the pricing measure $\mathbb{Q}$ of the four terms above. The four expectations are derived in detail in Appendix A.

Based on (3.4), we derive the Delta and cross-Gamma hedging parameters, which can be straightforwardly calculated by partial differentiation of the price $C_{t}$ with respect to the futures prices. All hedging parameters are given by the current futures price of the two underlying contracts and are therefore simple to implement in practice. The Delta hedge with respect to the energy futures is given by

$$
\begin{aligned}
\frac{\partial C_{t}}{\partial F_{t}^{E}\left(\tau_{1}, \tau_{2}\right)}= & F_{t}^{I}\left(\tau_{1}, \tau_{2}\right) \exp \left(-r\left(\tau_{2}-t\right)+\rho_{X, Y} \sigma_{X} \sigma_{Y}\right) \\
& \times\left(M\left(y_{1}^{* * *}, y_{2}^{* * *} ; \rho_{X, Y}\right)+B\left(y_{1}^{* * *}\right) N\left(y_{2}^{* * *}-\rho_{X, Y}\right) \frac{1}{\sigma_{X}}\right) \\
& -\bar{K}_{I} \mathrm{e}^{-r\left(\tau_{2}-t\right)}\left(M\left(y_{1}^{* *}, y_{2}^{* *} ; \rho_{X, Y}\right)+B\left(y_{1}^{* *}\right) N\left(y_{2}^{* *}-\rho_{X, Y}\right) \frac{1}{\sigma_{X}}\right) \\
& -\frac{F_{t}^{I}\left(\tau_{1}, \tau_{2}\right) \bar{K}_{E}}{F_{t}^{E}\left(\tau_{1}, \tau_{2}\right) \sigma_{X}} \mathrm{e}^{-r\left(\tau_{2}-t\right)} B\left(y_{1}^{*}\right) N\left(y_{2}^{*}-\rho_{X, Y}\right) \\
& +\frac{\bar{K}_{E} \bar{K}_{I}}{F_{t}^{E}\left(\tau_{1}, \tau_{2}\right) \sigma_{X}} \mathrm{e}^{-r\left(\tau_{2}-t\right)} B\left(y_{1}\right) N\left(y_{2}-\rho_{X, Y}\right)
\end{aligned}
$$

where $N(\cdot)$ denotes the standard normal cumulative distribution function, and

$$
B(x)=\frac{\mathrm{e}^{\left(x^{2}-\rho_{X, Y}^{2}\right)}}{4 \pi^{2}\left(1-\rho_{X, Y}^{2}\right)} .
$$

The Delta hedge with respect to the temperature index futures is of course analogous to the energy Delta hedge, only with the substitutions $F_{t}^{E}\left(\tau_{1}, \tau_{2}\right)=F_{t}^{I}\left(\tau_{1}, \tau_{2}\right)$, $y_{1}^{* * *}=y_{2}^{* * *}, y_{1}^{* *}=y_{2}^{* *}, y_{1}^{*}=y_{2}^{*}, y_{1}=y_{2}, \sigma_{Y}=\sigma_{X}$ and $\sigma_{X}=\sigma_{Y}$. The cross- 
Gamma hedge is given by

$$
\begin{aligned}
& \frac{\partial C_{t}^{2}}{\partial F_{t}^{E}\left(\tau_{1}, \tau_{2}\right) \partial F_{t}^{I}\left(\tau_{1}, \tau_{2}\right)} \\
&=\exp \left(-r\left(\tau_{2}-t\right)+\rho_{X, Y} \sigma_{X} \sigma_{Y}\right) \\
& \quad \times\left(M\left(y_{1}^{* * *}, y_{2}^{* * *} ; \rho_{X, Y}\right)+B\left(y_{2}^{* * *}\right) N\left(y_{1}^{* * *}-\rho_{X, Y}\right) \frac{1}{\sigma_{Y}}\right) \\
&+\exp \left(-r\left(\tau_{2}-t\right)+\rho_{X, Y} \sigma_{X} \sigma_{Y}\right) \\
& \quad \times B\left(y_{1}^{* * *}\right)\left(N\left(y_{2}^{* * *}-\rho_{X, Y}\right) \frac{1}{\sigma_{X}}+n\left(y_{2}^{* * *}-\rho_{X, Y}\right) \frac{1}{\sigma_{Y}}\right) \\
&-\frac{\bar{K}_{I}}{F_{t}^{I}\left(\tau_{1}, \tau_{2}\right) \sigma_{Y}} \mathrm{e}^{-r\left(\tau_{2}-t\right)} \\
& \quad \times\left(B\left(y_{2}^{* *}\right) N\left(y_{1}^{* *}-\rho_{X, Y}\right)+B\left(y_{1}^{* *}\right) n\left(y_{2}^{* *}-\rho_{X, Y}\right) \frac{1}{\sigma_{X}}\right) \\
&-\frac{\bar{K}_{E}}{F_{t}^{E}\left(\tau_{1}, \tau_{2}\right) \sigma_{X}} \mathrm{e}^{-r\left(\tau_{2}-t\right)} B\left(y_{1}^{*}\right)\left(N\left(y_{2}^{*}-\rho_{X, Y}\right)+n\left(y_{2}^{*}-\rho_{X, Y}\right) \frac{1}{\sigma_{Y}}\right) \\
&+ \frac{\bar{K}_{E} \bar{K}_{I}}{F_{t}^{E}\left(\tau_{1}, \tau_{2}\right) F_{t}^{I}\left(\tau_{1}, \tau_{2}\right)\left(\sigma_{X}+\sigma_{Y}\right)} \mathrm{e}^{-r\left(\tau_{2}-t\right)} B\left(y_{1}\right) n\left(y_{2}-\rho_{X, Y}\right), \quad(3.6)
\end{aligned}
$$

where $n(\cdot)$ denotes the standard normal probability density function (pdf). In our model, it is possible to hedge the quanto option perfectly, with positions described above by the three Delta and Gamma parameters. In practice, however, this would be difficult due to low liquidity in, for example, the temperature market. Further, as discussed in Section 2.2, we cannot trade futures in all markets within the delivery period, which puts additional restrictions on the suitability of the hedge. In such cases, the parameters above will guide in a partial hedging of the option.

\subsection{Two-dimensional Schwartz-Smith model with seasonality}

The popular commodity price model proposed by Schwartz and Smith (2000) is a natural starting point for deriving dynamics of energy futures. In this model, the logspot price is the sum of two processes, one representing the long-term dynamics of the commodity prices in the form of an arithmetic Brownian motion and one representing the short-term deviations from the long-run dynamics in the form of an OrnsteinUhlenbeck process, with a mean reversion level of zero. Other papers such as Lucia and Schwartz (2002) and Sørensen (2002) use the same two driving factors and extend the model to include seasonality. We choose the seasonality parameterization of the latter and further extend to a two-asset framework by linking the driving Brownian 
motions. The dynamics under $\mathbb{P}$ is given by

$$
\begin{aligned}
\log S_{t} & =\Lambda(t)+X_{t}+Z_{t}, \\
\mathrm{~d} X_{t} & =\left(\mu-\frac{1}{2} \sigma^{2}\right) \mathrm{d} t+\sigma \mathrm{d} \tilde{W}_{t}, \\
\mathrm{~d} Z_{t} & =-\kappa Z_{t} \mathrm{~d} t+v \mathrm{~d} \tilde{B}_{t} .
\end{aligned}
$$

Here, $\tilde{B}$ and $\tilde{W}$ are correlated Brownian motions and $\mu, \sigma, \kappa$ and $\eta$ are constants. The deterministic function $\Lambda(t)$ describes the seasonality of the log-spot prices. In order to price a futures contract written on an underlying asset with the above dynamics, a measure change from $\mathbb{P}$ to an equivalent probability $\mathbb{Q}$ is made:

$$
\begin{aligned}
& \mathrm{d} X_{t}=\left(\alpha-\frac{1}{2} \sigma^{2}\right) \mathrm{d} t+\sigma \mathrm{d} W_{t}, \\
& \mathrm{~d} Z_{t}=-\left(\lambda_{Z}+\kappa Z_{t}\right) \mathrm{d} t+v \mathrm{~d} B_{t}^{i} .
\end{aligned}
$$

Here, $\alpha=\mu-\lambda_{X}$, and $\lambda_{X}$ and $\lambda_{Z}$ are constant market prices of risk associated with $X_{t}$ and $Z_{t}$ for asset $i$, respectively. This corresponds to a Girsanov transformation of $\tilde{B}$ and $\tilde{W}$ by a constant drift, so that $B$ and $W$ become two correlated $\mathbb{Q}$-Brownian motions. As is well-known for the Girsanov transformation, the correlation between $B$ and $W$ is the same under $\mathbb{Q}$ as that for $\tilde{B}$ and $\tilde{W}$ under $\mathbb{P}$ (see Karatzas and Shreve 2000). Following Sørensen (2002), the futures price $F_{t}(\tau)$ at time $t \geqslant 0$ of a contract with delivery at time $\tau \geqslant t$ has the following form on a log scale (note that it is the Schwartz-Smith futures price scaled by a seasonality function):

$$
\log F_{t}(\tau)=\Lambda(\tau)+A(\tau-t)+X_{t}+Z_{t} \mathrm{e}^{-\kappa(\tau-t)},
$$

where

$$
A(\tau)=\alpha \tau-\frac{\lambda_{Z}-\rho \sigma \nu}{\kappa}\left(1-\mathrm{e}^{-\kappa \tau}\right)+\frac{\nu^{2}}{4 \kappa}\left(1-\mathrm{e}^{-2 \kappa \tau}\right) .
$$

The $\log$ futures prices are affine in the two factors $X$ and $Z$ driving the spot price and scaled by functions of time to delivery $\tau-t$ and by functions of time of delivery $\tau$. Sørensen (2002) chooses to parameterize the seasonality function $\Lambda$ by a linear combination of cosine and sine functions:

$$
\Lambda(t)=\sum_{k=1}^{K}\left(\gamma_{k} \cos (2 \pi k t)+\gamma_{k}^{*} \sin (2 \pi k t)\right) .
$$

In this paper, we have highlighted the fact that the payoff of energy quanto options can be expressed in terms of the futures prices of energy and temperature indexes. We may use the above procedure to derive futures price dynamics from a model of the spot. However, we may also directly state a futures price dynamics in the fashion of Heath, Jarrow and Morton, using the above model as inspiration for the specification of the 
model. The HJM approach was proposed to model energy futures by Clewlow and Strickland (2000), and it was later investigated in detail by Benth and Koekebakker (2008) (see also Benth et al 2008; Miltersen and Schwartz 1998). We follow this approach here, proposing a joint model for the energy and temperature index futures price based on the seasonal Schwartz-Smith model.

In stating such a model, we must account for the fact that the futures in question are delivering over a period $\left[\tau_{1}, \tau_{2}\right]$, and not at a fixed delivery time $\tau$. An attractive alternative to the additive approach by Lucia and Schwartz (2002) is to let $F_{t}\left(\tau_{1}, \tau_{2}\right)$ itself follow a dynamics of the form (3.7), with some appropriately chosen dependency on $\tau_{1}$ and $\tau_{2}$. For example, we may choose $\tau=\tau_{1}$ in (3.7), or $\tau=\left(\tau_{1}+\tau_{2}\right) / 2$, or any other time within the delivery period $\left[\tau_{1}, \tau_{2}\right]$. In this way, we will account for the delivery-time effect in the futures price dynamics, sometimes referred to as the Samuelson effect. We remark that it is well-known that, for futures delivering over a certain period, the volatility will not converge to that of the underlying spot as time to delivery goes to zero (see Benth et al 2008). Through the above parameter choices, we obtain such an effect.

In order to jointly model the energy and temperature futures prices, two futures dynamics of the type in (3.7) are connected by allowing the Brownian motions to be correlated across assets. We will have four Brownian motions $W^{E}, B^{E}, W^{I}$ and $B^{I}$ in our two-asset, two-factor model. These are assumed to be correlated as follows: $\rho_{E}=\operatorname{corr}\left(W_{1}^{E}, B_{1}^{E}\right), \rho_{I}=\operatorname{corr}\left(W_{1}^{I}, B_{1}^{I}\right), \rho_{W}=\operatorname{corr}\left(W_{1}^{E}, W_{1}^{I}\right)$ and $\rho_{B}=\operatorname{corr}\left(B_{1}^{E}, B_{1}^{I}\right)$. Moreover, we have cross-correlations given by

$$
\begin{aligned}
& \rho_{I, E}^{W, B}=\operatorname{corr}\left(W_{1}^{I}, B_{1}^{E}\right), \\
& \rho_{E, I}^{W, B}=\operatorname{corr}\left(W_{1}^{E}, B_{1}^{I}\right) .
\end{aligned}
$$

In an HJM style, we assume that the joint dynamics of the futures price processes $F_{t}^{E}\left(\tau_{1}, \tau_{2}\right)$ and $F_{t}^{I}\left(\tau_{1}, \tau_{2}\right)$ under $\mathbb{Q}$ is given by

$$
\frac{\mathrm{d} F_{t}^{i}\left(\tau_{1}, \tau_{2}\right)}{F_{t}^{i}\left(\tau_{1}, \tau_{2}\right)}=\sigma_{i} \mathrm{~d} W_{t}^{i}+\eta_{i}(t) \mathrm{d} B_{t}^{i}
$$

for $i=E, I$, and with

$$
\eta_{i}(t)=v_{i} \mathrm{e}^{-\kappa^{i}\left(\tau_{2}-t\right)} .
$$

Note that we suppose the futures price is a martingale with respect to the pricing measure $\mathbb{Q}$, which is natural from the point of view that we want an arbitrage-free model. Moreover, we have made the explicit choice here that $\tau=\tau_{2}$ in (3.7) when modeling the delivery-time effect.

Note that

$$
\mathrm{d} \log F_{t}^{i}\left(\tau_{1}, \tau_{2}\right)=-\frac{1}{2}\left(\sigma_{i}^{2}+\eta_{i}(t)^{2}+2 \rho_{i} \sigma_{i} \eta_{i}(t)\right) \mathrm{d} t+\sigma_{i} \mathrm{~d} \tilde{W}_{t}^{i}+\eta_{i}(t) \mathrm{d} \tilde{B}_{t}^{i}
$$


for $i=E, I$. Hence, we can make the representation

$$
F_{T}^{E}\left(\tau_{1}, \tau_{2}\right)=F_{t}^{E}\left(\tau_{1}, \tau_{2}\right) \exp \left(-\mu_{E}+X\right)
$$

by choosing

$$
X \sim \mathcal{N}(0, \underbrace{\int_{t}^{T}\left(\sigma_{E}^{2}+\eta_{E}(s)^{2}+2 \rho_{E} \sigma_{E} \eta_{E}(s)\right) \mathrm{d} s}_{\sigma_{X}^{2}}), \quad \mu_{E}=-\frac{1}{2} \sigma_{X}^{2}
$$

and similarly for $F_{T}^{I}\left(\tau_{1}, \tau_{2}\right)$. These integrals can be computed analytically in the above model, where $\eta_{i}(t)=v_{i} \mathrm{e}^{-\kappa^{i}\left(\tau_{2}-t\right)}$. We can also compute the correlation $\rho_{X, Y}$ analytically, since

$$
\rho_{X, Y}=\frac{\operatorname{cov}(X, Y)}{\sigma_{X} \sigma_{Y}}
$$

and

$$
\begin{aligned}
\operatorname{cov}(X, Y)=\rho_{W} \int_{t}^{T} & \sigma_{E} \sigma_{I} \mathrm{~d} s+\rho_{E, I}^{W, B} \int_{t}^{T} \sigma_{E} \eta_{I}(s) \mathrm{d} s \\
& +\rho_{I, E}^{W, B} \int_{t}^{T} \eta_{E}(s) \sigma_{I} \mathrm{~d} s+\rho_{B} \int_{t}^{T} \eta_{E}(s) \eta_{I}(s) \mathrm{d} s .
\end{aligned}
$$

A closed-form expression of this covariance can be computed. In the special case of zero cross-correlations, this simplifies to

$$
\operatorname{cov}(X, Y)=\rho_{W} \int_{t}^{T} \sigma_{E} \sigma_{I} \mathrm{~d} s+\rho_{B} \int_{t}^{T} \eta_{E}(s) \eta_{I}(s) \mathrm{d} s
$$

The exact expressions for $\sigma_{X}, \sigma_{Y}$ and $\operatorname{cov}(X, Y)$ in the two-dimensional SchwartzSmith model with seasonality are presented in Appendix B.

This bivariate futures price model has a form that can be immediately used for pricing energy quanto options by inferring the result in Proposition 3.1. We shall come back to this model in the empirical case study in Section 4. The general setup in Section 3 includes the implied forward dynamics from general multifactor spot models, with stationary and nonstationary terms. Hence, this is a very general pricing mechanism, where the essential problem is to identify the overall volatilities $\sigma_{X}$ and $\sigma_{Y}$ and the cross-correlation $\rho_{X, Y}$. As a final remark, we note that our pricing approach only looks at futures dynamics up to the start of the delivery period $\tau_{1}$. As briefly discussed in Section 3.2, it is reasonable to expect that the dynamics of a futures contract should be different within the delivery period $\left[\tau_{1}, \tau_{2}\right]$. For times $t$ within $\left[\tau_{1}, \tau_{2}\right]$, we will, in the case of the energy futures, have

$$
F_{t}\left(\tau_{1}, \tau_{2}\right)=\frac{1}{\tau_{2}-\tau_{1}} \sum_{u=\tau_{1}}^{t} S_{u}+\mathbb{E}_{t}^{\mathbb{Q}}\left[\frac{1}{\tau_{2}-\tau_{1}} \sum_{u=t+1}^{\tau_{2}} S_{u}\right]
$$


FIGURE 1 The evolution of the gas futures curve as a function of maturity $\tau_{2}$.

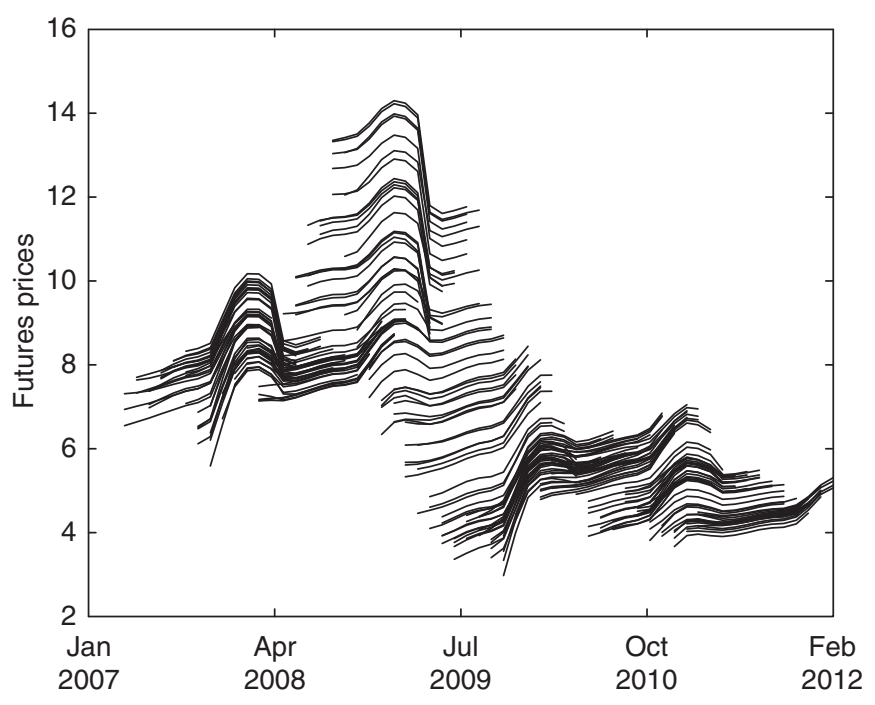

For each day $t$, the observed futures curve $F_{t}\left(\tau_{2}-\Delta, \tau_{2}\right)$ with $\Delta=1$ month is plotted as a function of $\tau_{2}$. We observe up to twelve maturities at each observation point $t$. From $t=$ January 1, 2007 to December 31, 2010 one observed futures curve per week is plotted.

Thus, the futures price must consist of two parts, the first simply the tracked observed energy spot up to time $t$, and the second the current futures price of a contract with delivery period $\left[t, \tau_{2}\right]$. This latter part will have a volatility that must go to zero as $t$ tends toward $\tau_{2}$.

\section{EMPIRICAL ANALYSIS}

In this section, we present an empirical study of energy quanto options written on NYMEX natural gas futures and the HDD temperature index. We present the futures price data, which constitutes the basis of our analysis, and estimate the parameters in the joint futures price model (3.9). We then discuss the impact of correlation on the valuation of the option to be priced.

\subsection{Data}

Futures contracts for the delivery of gas are traded on NYMEX monthly for ten years. The underlying is the delivery of gas throughout a month and the price is per unit. The contract trades until a couple of days before the delivery month. Many contracts are closed prior to the last trading day, and we choose the first twelve contracts for 
FIGURE 2 The evolution of the New York HDD futures curve as a function of maturity $\tau_{2}$.

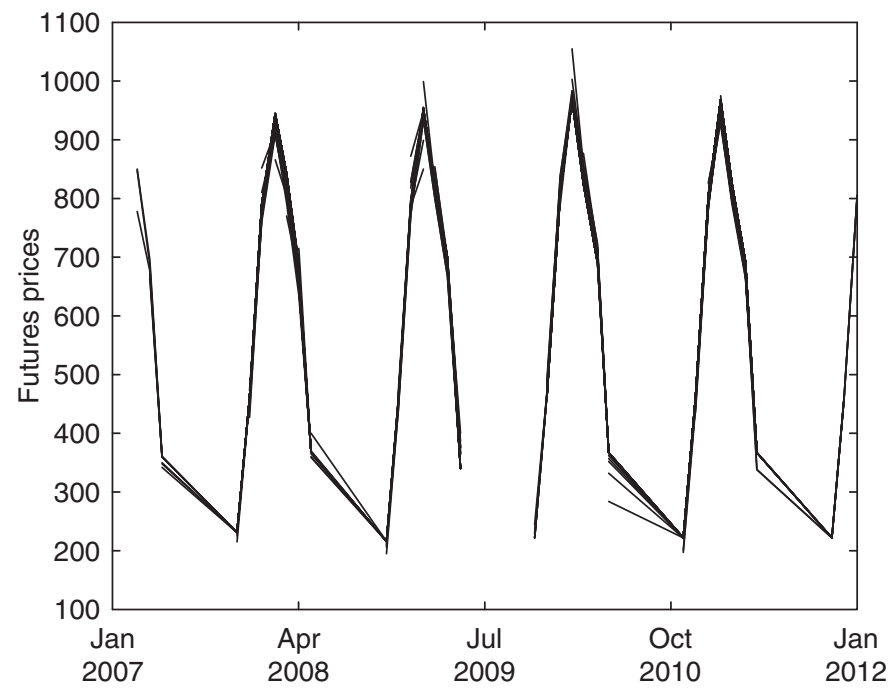

For each day $t$, the observed futures curve $F_{t}\left(\tau_{2}-\Delta, \tau_{2}\right)$ with $\Delta=1$ month is plotted as a function of $\tau_{2}$. We observe up to seven maturities at each observation point $t$. From $t=$ January 1, 2007 to December 31,2010 one observed futures curve per week is plotted. The number of curves is the same as in Figure 1 on the facing page, but, because of low liquidity, HDD futures prices do not fluctuate much from day to day, except for the first contracts. Therefore, many of the curves are superimposed.

delivery at least one month later, ie, for January 2, we use March 2007-February 2008 contracts. We denote the time $t$ futures price for a contract delivering one month $(=\Delta)$ until $\tau_{2}$ by $F_{t}^{E}\left(\left[\tau_{2}-\Delta, \tau_{2}\right]\right)$ and let the price follow a process of the type (3.7) discussed in Section 3.2. When investigating data, there is a seasonality pattern over the year, where prices are, in general, lowest in late spring and early fall, slightly higher in between these periods and highest in the winter. These two "peaks" during the year are modeled by setting $K=2$ in (3.8) similar to the seasonal pattern of the commodities studied in Sørensen (2002). They are supported by the statistical significance of the parameter estimates and standard errors for the $\gamma$ s. The evolution of the futures gas curves is shown in Figure 1 on the facing page.

Futures contracts on accumulated HDD are traded on the CME for several cities for October, November, December, January, February, March and April, a couple of years out. The contract value is US\$20 for the number of HDD accumulated over the month for a specific location, ie, a day with temperature $60^{\circ} \mathrm{F}$ adds 5 to the index and thereby US\$100 to the final settlement, whereas a day with temperature $70^{\circ} \mathrm{F}$ does not add to or subtract from the index. The contract trades until the beginning of the concurrent month. The futures price is denoted by $F_{t}^{I}\left(\tau_{2}-\Delta, \tau_{2}\right)$ and 
settled on the accumulated index, $\sum \mathrm{HDD}_{u}$. Liquidity is basically nonexistent after the first year, so for every day we choose the first seven contracts where the index period has not yet started, ie, for January 2, 2007 we use the February 2007, March 2007, April 2007, October 2007, November 2007, December 2007 and January 2008 contracts.

Again, we let the futures price follow a price process of the type (3.7). The stationary part represents the short-term random fluctuations in the underlying temperature deviation. Over a long time, we might argue that temperature and thereby a month of accumulated HDD has a long-term drift, but, during the time period our data covers, the effect of long-term environmental changes is negligible. The short time period covered justifies leaving out the nonstationary part, $X$. However, estimation of the full model led to significant parameter estimates for $\sigma_{I}$ (see Section 4.2 for estimation results), so we choose to keep the long-term component in for the temperature index as well.

Inspection of the data makes it clear that there is a deterministic level for each month, which does not change much until we get close to the index period and the weather reports start to add information and affect prices. An obvious choice for modeling this deterministic seasonal component can be found in Lucia and Schwartz (2002), where the seasonality is modeled by a dummy for each month. With seven observed contracts, this would give us four additional parameters to estimate. Due to this, and to keep the two models symmetrical, we choose to keep the same structure as for the gas, but with $K=1$ in (3.8). The chosen locations are New York and Chicago, due to their being areas with fairly large gas consumption. The development in the term structure of HDD futures prices for New York is shown in Figure 2 on the preceding page, where the daily observed futures curves are plotted as a function of $\tau_{2}$.

\subsection{Estimation results}

We estimate the parameters using maximum likelihood estimation via the Kalman filter technique (see Appendix D), as in Sørensen (2002). The resulting parameter estimates are reported in Table 2 on the facing page for the joint modeling of gas futures and New York HDD futures and in Table 3 on page 28 for the joint modeling of gas futures and Chicago HDD futures, with standard errors based on the Hessian of the loglikelihood function given in parentheses.

Both under the physical and the risk-neutral measure, the drift of the long-term component for gas is negative. This matches the decrease in gas prices over time. The volatility parameters correspond to a term structure of volatility that for gas starts at around $50 \%$. For the HDD futures, the annualized volatility starts at a very high level of more than $100 \%$ for the closest contract and then quickly drops. For both types of 
TABLE 2 Parameter estimates for the two-dimensional, two-factor model with seasonality when New York HDD futures and NYMEX gas futures are modeled jointly.

\begin{tabular}{|c|c|c|}
\hline & HDD (NY) & Gas \\
\hline$\mu$ & $\begin{array}{c}0.0063 \\
(0.0247)\end{array}$ & $\begin{array}{r}-0.0850 \\
(0.0989)\end{array}$ \\
\hline$\kappa$ & $\begin{array}{l}16.5654 \\
(1.1023)\end{array}$ & $\begin{array}{c}0.6116 \\
(0.0320)\end{array}$ \\
\hline$\sigma$ & $\begin{array}{c}0.0494 \\
(0.0059)\end{array}$ & $\begin{array}{c}0.2342 \\
(0.0200)\end{array}$ \\
\hline$v$ & $\begin{array}{c}3.6517 \\
(0.6197)\end{array}$ & $\begin{array}{c}0.6531 \\
(0.0332)\end{array}$ \\
\hline$\rho$ & $\begin{array}{c}-0.6066 \\
(0.0801)\end{array}$ & $\begin{array}{r}-0.6803 \\
(0.0656)\end{array}$ \\
\hline$\alpha$ & $\begin{array}{c}0.0027 \\
(0.0049)\end{array}$ & $\begin{array}{r}-0.3366 \\
(0.0246)\end{array}$ \\
\hline$\lambda$ & $\begin{array}{l}-5.9581 \\
(2.3059)\end{array}$ & $\begin{array}{r}-0.9191 \\
(0.1968)\end{array}$ \\
\hline$\sigma_{\epsilon}$ & $\begin{array}{c}0.0655 \\
(0.0006)\end{array}$ & $\begin{array}{c}0.0199 \\
(0.0001)\end{array}$ \\
\hline$\gamma_{1}$ & $\begin{array}{c}0.9044 \\
(0.0023)\end{array}$ & $\begin{array}{c}0.0500 \\
(0.0003)\end{array}$ \\
\hline$\gamma_{1}^{*}$ & $\begin{array}{c}0.8104 \\
(0.0018)\end{array}$ & $\begin{array}{c}0.0406 \\
(0.0003)\end{array}$ \\
\hline$\gamma_{2}$ & N/A & $\begin{array}{c}0.0128 \\
(0.0003)\end{array}$ \\
\hline$\gamma_{2}^{*}$ & $\mathrm{~N} / \mathrm{A}$ & $\begin{array}{c}0.0270 \\
(0.0003)\end{array}$ \\
\hline$\rho^{W}$ & \multicolumn{2}{|c|}{$\begin{array}{c}-0.2843 \\
(0.0904)\end{array}$} \\
\hline$\rho^{B}$ & \multicolumn{2}{|c|}{$\begin{array}{c}0.1817 \\
(0.0678)\end{array}$} \\
\hline$\ell$ & \multicolumn{2}{|c|}{36198} \\
\hline
\end{tabular}

contracts, we see a negative correlation between the long- and short-term factors. For gas, this is obvious, because it creates a mean reversion effect that is characteristic of commodities. The positive short-term correlation reflects the connection between temperature and prices. If there is a short-term shock in temperature, this is reflected in the closest HDD futures contract. At the same time, there is an increase in demand for gas, which leads to a short-term increase in gas prices. The standard deviation of the estimation error for the log prices is, on average, $2 \%$ for the gas contracts and a bit higher (around 6\%) for the HDD contracts. Figure 3 on the next page shows the 
FIGURE 3 Model prices (solid black line) and observed prices (dashed gray line) for the closest maturity when prices of natural gas futures and New York HDD futures are modeled jointly.

(a)

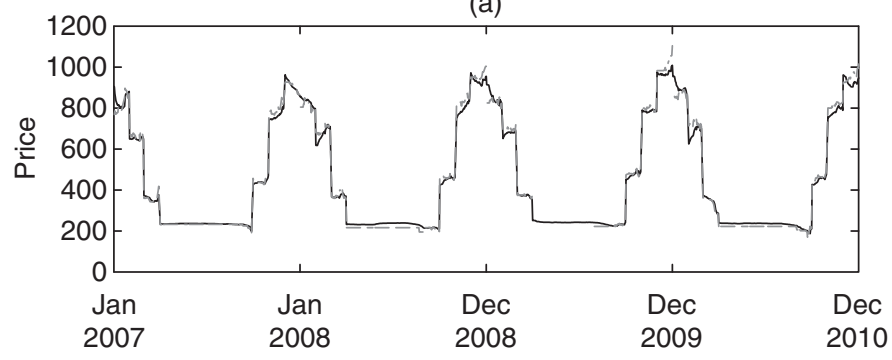

(b)

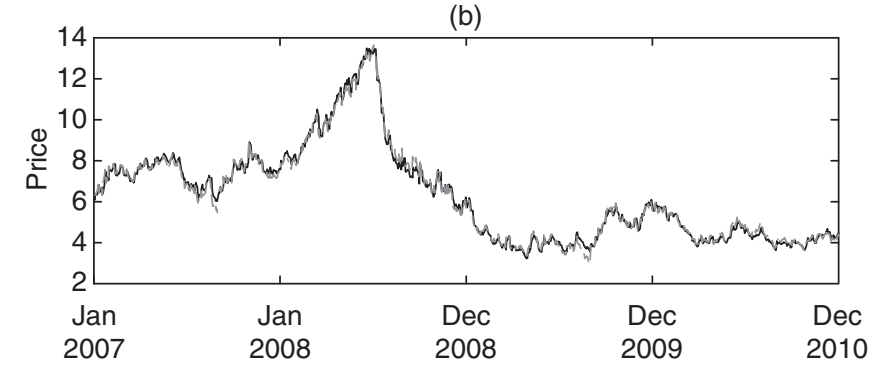

(a) Closest HDD. (b) Closest gas futures. The error between model and observed prices has a standard deviation of around $2 \%$, respectively $6.5 \%$. For the HDD futures contracts especially, the roll time of the futures contract is identifiable by the jump in prices. For the period April-September, the closest HDD future is the October contract, which can be seen in the figure as the longer, flatter lines.

model fit along with observed data and Figure 4 on the facing page and Figure 5 on page 22 show the squared pricing errors.

\subsection{A case study}

To consider the impact of the connection between gas prices and temperature (and thus gas and HDD futures), we compare the quanto option prices with prices obtained under the assumption of independence, and thus priced using the model in Black (1976) (see Appendix C). If the two futures were independent, we would get $\left(C_{t}^{0}\right.$ denotes the price under the zero-correlations assumption)

$$
C_{t}^{0}=\mathrm{e}^{-r\left(\tau_{2}-t\right)} \mathbb{E}_{t}^{Q}\left[\max \left(F_{\tau_{2}}^{E}\left(\tau_{1}, \tau_{2}\right)-\bar{K}_{E}, 0\right)\right] \mathbb{E}_{t}^{Q}\left[\max \left(F_{\tau_{2}}^{I}\left(\tau_{1}, \tau_{2}\right)-\bar{K}_{I}, 0\right)\right],
$$

which can be viewed as the product of the prices of two plain-vanilla call options on the gas and HDD futures, respectively. In fact, we have the price $C_{t}^{0}$ given in this case as the product of two Black (1976) formulas using the interest rate $r / 2$ in the two 
FIGURE 4 The time series of squared errors of the percentage differences between fitted and actual New York HDD futures prices when modeled jointly with NYMEX natural gas futures.
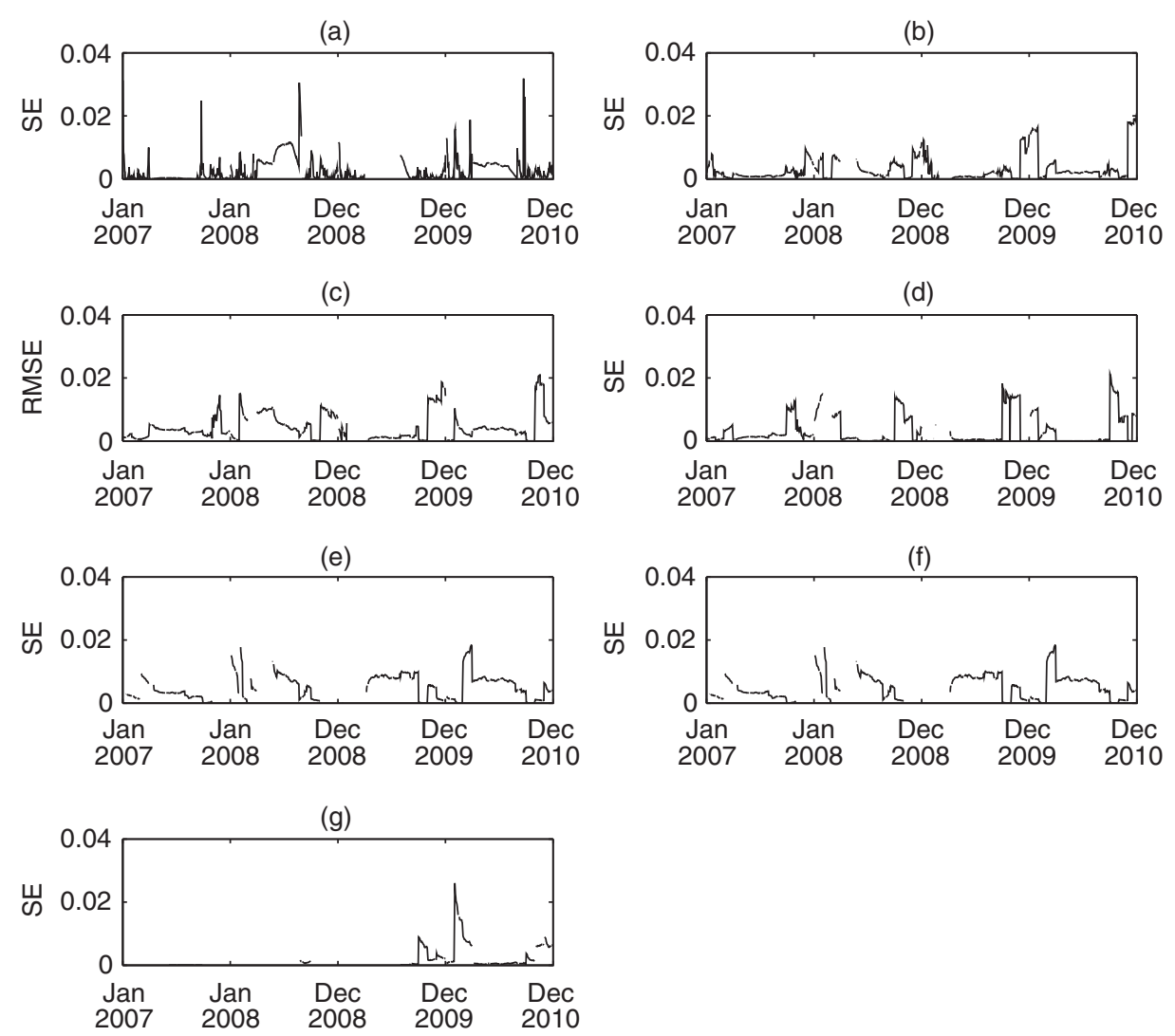

(a) Closest. (b) Second closest. (c) Third closest. (d) Fourth closest. (e) Fifth closest. (f) Sixth closest. (g) Seventh closest. The pricing errors jump when the contracts roll.

respective prices. From Figure 6 on page 23 and Figure 7 on page 23, it is clear that the correlation between the gas and HDD futures significantly impacts the quanto option price. Part (a) of Figure 6 and part (a) of Figure 7 show the quanto option price on December 31, 2010 for settlement months December 2011 and February 2011, respectively. Part (b) of both Figure 6 and Figure 7 shows the relative pricing error between the quanto option price with and without correlation across assets. The ratio of the change in quanto option price to the product of the marginal options, ie, $\left(C_{t}-C_{t}^{0}\right) / C_{t}$, is plotted. For a short time to maturity, we see a relative pricing error of more than $75 \%$ for the high strikes. The fact that the observed correlation increases the 
FIGURE 5 The time series of squared errors of the percentage differences between fitted and actual natural gas futures prices when modeled jointly with New York HDD futures.

(a)

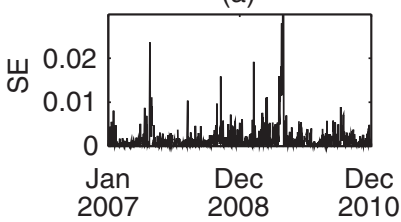

(d)

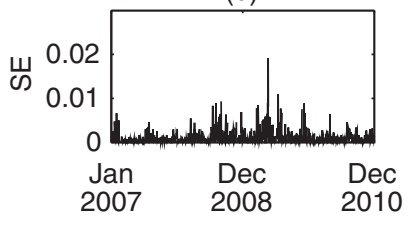

(g)

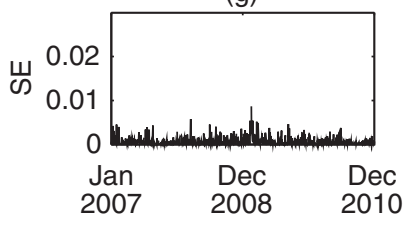

(j)

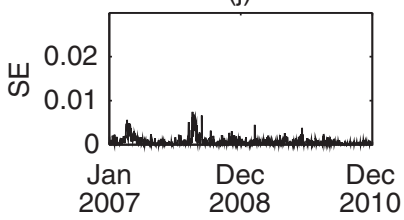

(b)

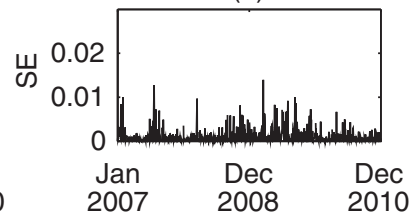

(e)

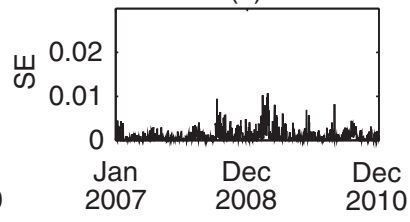

(h)

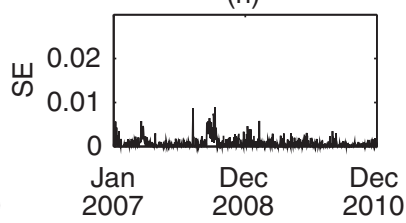

(k)

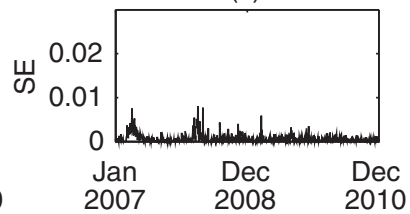

(c)

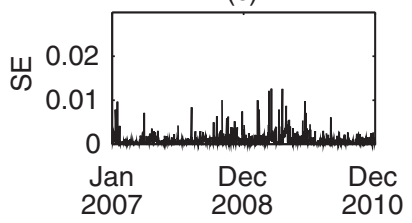

(f)

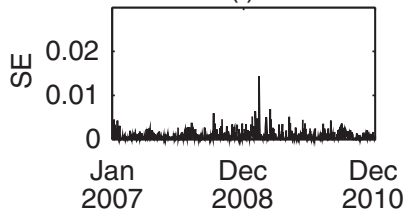

(i)

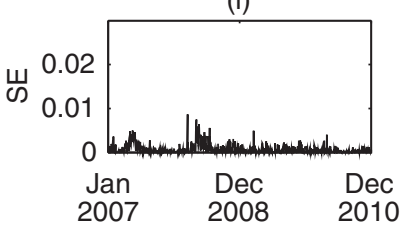

(I)

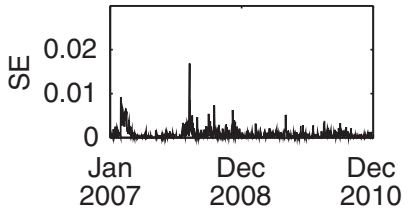

(a) Closest. (b) Second closest. (c) Thirrd closest. (d) Fourth closest. (e) Fifth closest. (f) Sixth closest. (g) Seventh closest. (h) Eighth closest. (i) Ninth closest. (j) Tenth closest. (k) Eleventh closest. (I) Twelfth closest. The pricing errors are largest around the 2008 boom/bust in energy prices.

quanto option price compared with the product of the two marginal options indicates that more probability mass lies in the quanto's exercise region. For short times to maturity especially, ignoring correlation can lead to significant underpricing of the quanto option.

\section{CONCLUDING REMARKS}

In this paper, we presented a closed-form pricing formula for an energy quanto option under the assumption that the underlying assets were lognormal. Taking advantage of the fact that energy and temperature futures are designed with a delivery period, 
FIGURE 6 Quanto option prices for a one-year option and relative pricing error compared with no correlation across assets.

(a)

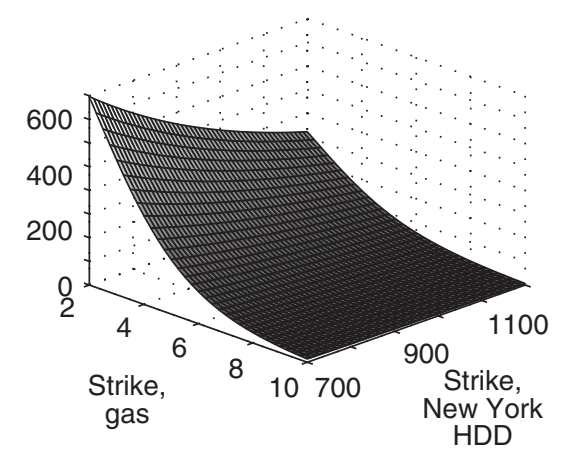

(b)

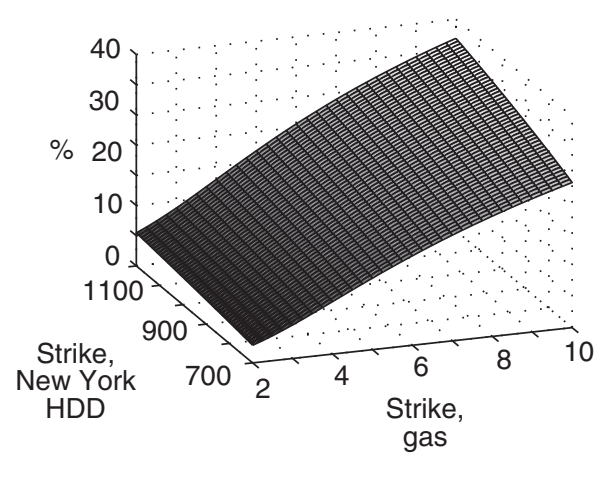

(a) The price of a quanto option as a function of the two strike values. The contract is priced on December 31,2010 for settlement in December 2011. Quanto price, $r=0.02, \tau_{1}=$ December 1, 2011, $\tau_{2}=$ December 31, 2011, $t=$ Dec 31, 2010. (b) The relative pricing error between the quanto option price calculated with and without correlation across assets. The interest rate is set to $2 \%$. Current futures prices are 5.0920 and 805 , respectively. Depending on the strikes, the relative price error is up to $40 \%$.

FIGURE 7 Quanto option prices for a one-month option and relative pricing error compared with no correlation across assets.

(a)

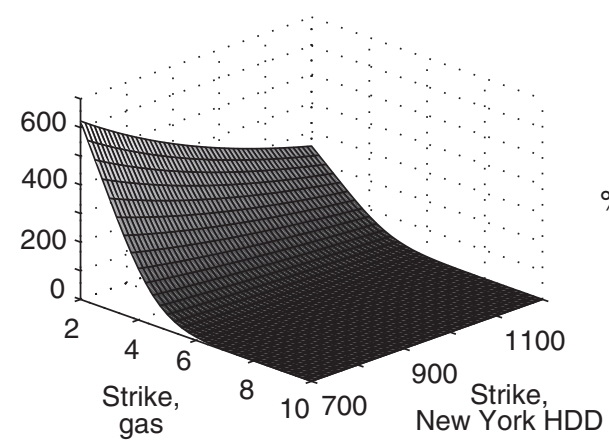

(b)

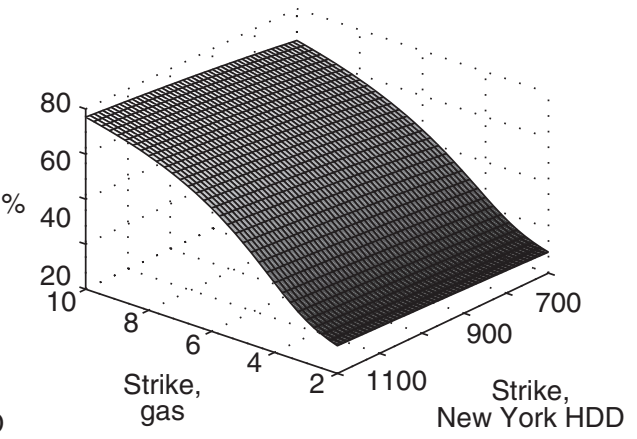

(a) The price of a quanto option as a function of the two strike values. The contract is priced on December 31,2010 for settlement in February 2011. (b) The relative pricing error between the quanto option price calculated with and without correlation across assets. The interest rate is set to $2 \%$. Current futures prices are 4.405 , respectively 797 . Depending on the strikes, the relative price error can be more than $75 \%$. 
FIGURE 8 The evolution of the Chicago HDD futures curve as a function of maturity $\tau_{2}$.

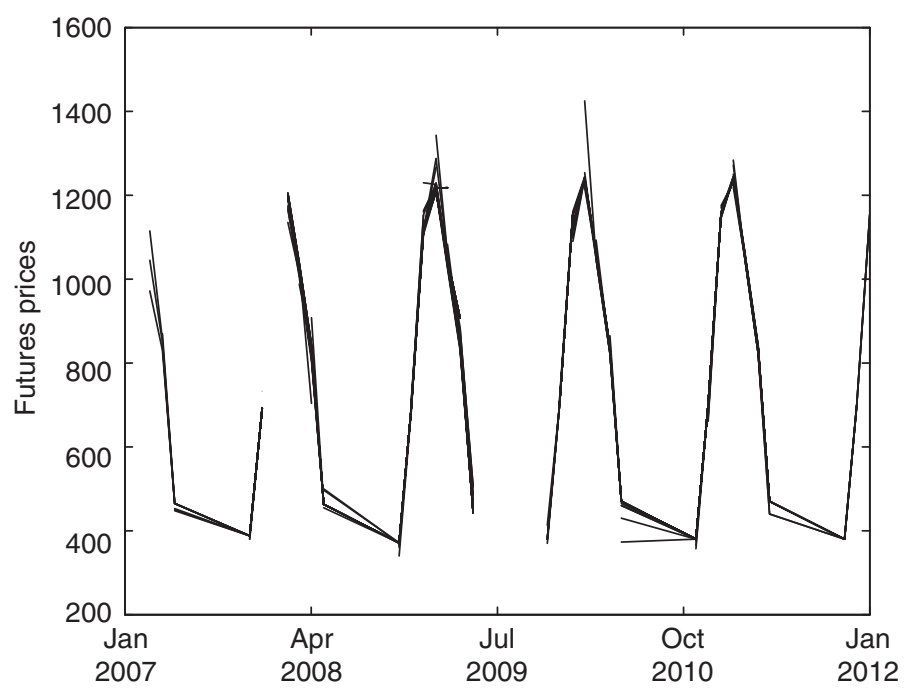

For each day $t$, the observed futures curve $F_{t}\left(\tau_{2}-\Delta, \tau_{2}\right)$ with $\Delta=1$ month is plotted as a function of $\tau_{2}$. We observe up to seven maturities at each observation point $t$. From $t=$ January 1, 2007 to December 31,2010 one observed futures curve per week is plotted. The number of curves is the same as in Figure 1 on page 16, but because of low liquidity HDD futures prices do not fluctuate much from day to day except for the first contracts.

we showed how quanto options can be priced using futures contracts as underlying assets. Correspondingly, we adopted an HJM approach and modeled the dynamics of the futures contracts directly. We showed that our approach encompasses relevant cases, such as GBMs and multifactor spot models. Importantly, our approach enabled us to derive hedging strategies and perform hedges with traded assets. We illustrated the use of our pricing model by estimating a two-dimensional, two-factor model with seasonality using NYMEX data on natural gas and CME data on temperature HDD futures. We calculated quanto energy option prices and showed how correlation between the two asset classes significantly impacts the prices.

\section{APPENDIX A. PROOF OF PRICING FORMULA}

In Section 3.1, we showed that the payoff function in (2.6) could be rewritten in the following way:

$$
\begin{aligned}
\hat{p}\left(F_{T}^{E}, F_{T}^{I}, \bar{K}_{I}, \bar{K}_{E}\right) & \\
& =\max \left(F_{T}^{I}-\bar{K}_{I}, 0\right) \max \left(F_{T}^{E}-\bar{K}_{E}, 0\right)
\end{aligned}
$$


FIGURE 9 The model prices (black solid line) and observed prices (dashed gray line) when prices of natural gas futures and Chicago HDD futures are modeled jointly.
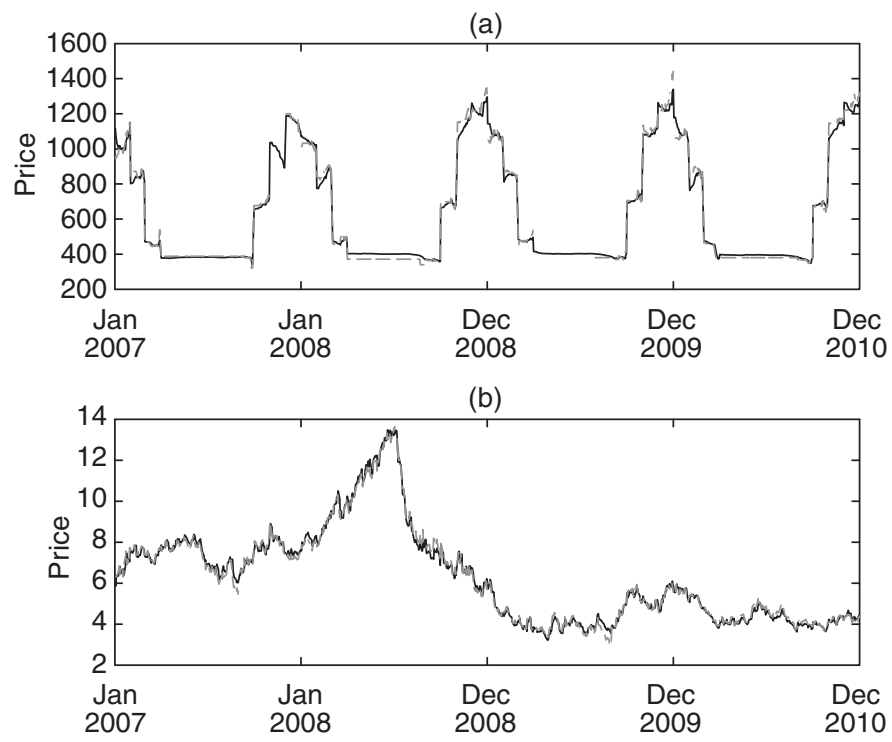

(a) Closest HDD. (b) Closest gas futures. The error between model and observed prices has a standard deviation of around $2 \%$, respectively $5.5 \%$.

$$
\begin{aligned}
= & \left(F_{T}^{E}-\bar{K}_{E}\right)\left(F_{T}^{I}-\bar{K}_{I}\right) 1_{\left\{F_{T}^{E}>\bar{K}_{E}\right\}} 1_{\left\{F_{T}^{I}>\bar{K}_{I}\right\}} \\
= & F_{T}^{E} F_{T}^{I} 1_{\left\{F_{T}^{E}>\bar{K}_{E}\right\}} 1_{\left\{F_{T}^{I}>\bar{K}_{I}\right\}}-F_{T}^{E} \bar{K}_{I} 1_{\left\{F_{T}^{E}>\bar{K}_{E}\right\}} 1_{\left\{F_{T}^{I}>\bar{K}_{I}\right\}} \\
& -F_{T}^{I} \bar{K}_{E} 1_{\left\{F_{T}^{E}>\bar{K}_{E}\right\}} 1_{\left\{F_{T}^{I}>\bar{K}_{I}\right\}}+\bar{K}_{E} \bar{K}_{I} 1_{\left\{F_{T}^{E}>\bar{K}_{E}\right\}} 1_{\left\{F_{T}^{I}>\bar{K}_{I}\right\}} .
\end{aligned}
$$

Now, let us calculate the expectation under $\mathbb{Q}$ of the payoff function, ie,

$$
\mathbb{E}_{t}^{\mathbb{Q}}\left[\hat{p}\left(F_{T}^{E}, F_{T}^{I}, \bar{K}_{I}, \bar{K}_{E}\right)\right] .
$$

We have

$$
\begin{aligned}
\mathbb{E}_{t}^{\mathbb{Q}}[ & \left.\hat{p}\left(F_{T}^{E}, F_{T}^{I}, \bar{K}_{I}, \bar{K}_{E}\right)\right] \\
= & \mathbb{E}_{t}^{\mathbb{Q}}\left[\max \left(F_{T}^{I}-\bar{K}_{I}, 0\right) \max \left(F_{T}^{E}-\bar{K}_{E}, 0\right)\right] \\
= & \mathbb{E}_{t}^{\mathbb{Q}}\left[F_{T}^{E} F_{T}^{I} 1_{\left\{F_{T}^{E}>\bar{K}_{E}\right\}} 1_{\left\{F_{T}^{I}>\bar{K}_{I}\right\}}\right]-\mathbb{E}_{t}^{\mathbb{Q}}\left[F_{T}^{E} \bar{K}_{I} 1_{\left\{F_{T}^{E}>\bar{K}_{E}\right\}} 1_{\left\{F_{T}^{I}>\bar{K}_{I}\right\}}\right] \\
& \quad-\mathbb{E}_{t}^{\mathbb{Q}}\left[F_{T}^{I} \bar{K}_{E} 1_{\left\{F_{T}^{E}>\bar{K}_{E}\right\}} 1_{\left\{F_{T}^{I}>\bar{K}_{I}\right\}}\right]+\mathbb{E}_{t}^{\mathbb{Q}}\left[\bar{K}_{E} \bar{K}_{I} 1_{\left\{F_{T}^{E}>\bar{K}_{E}\right\}} 1_{\left\{>\bar{K}_{I}\right\}}\right] .
\end{aligned}
$$


FIGURE 10 The time series of squared errors of the percentage differences between fitted and actual Chicago HDD futures prices, when modeled jointly with NYMEX natural gas futures.
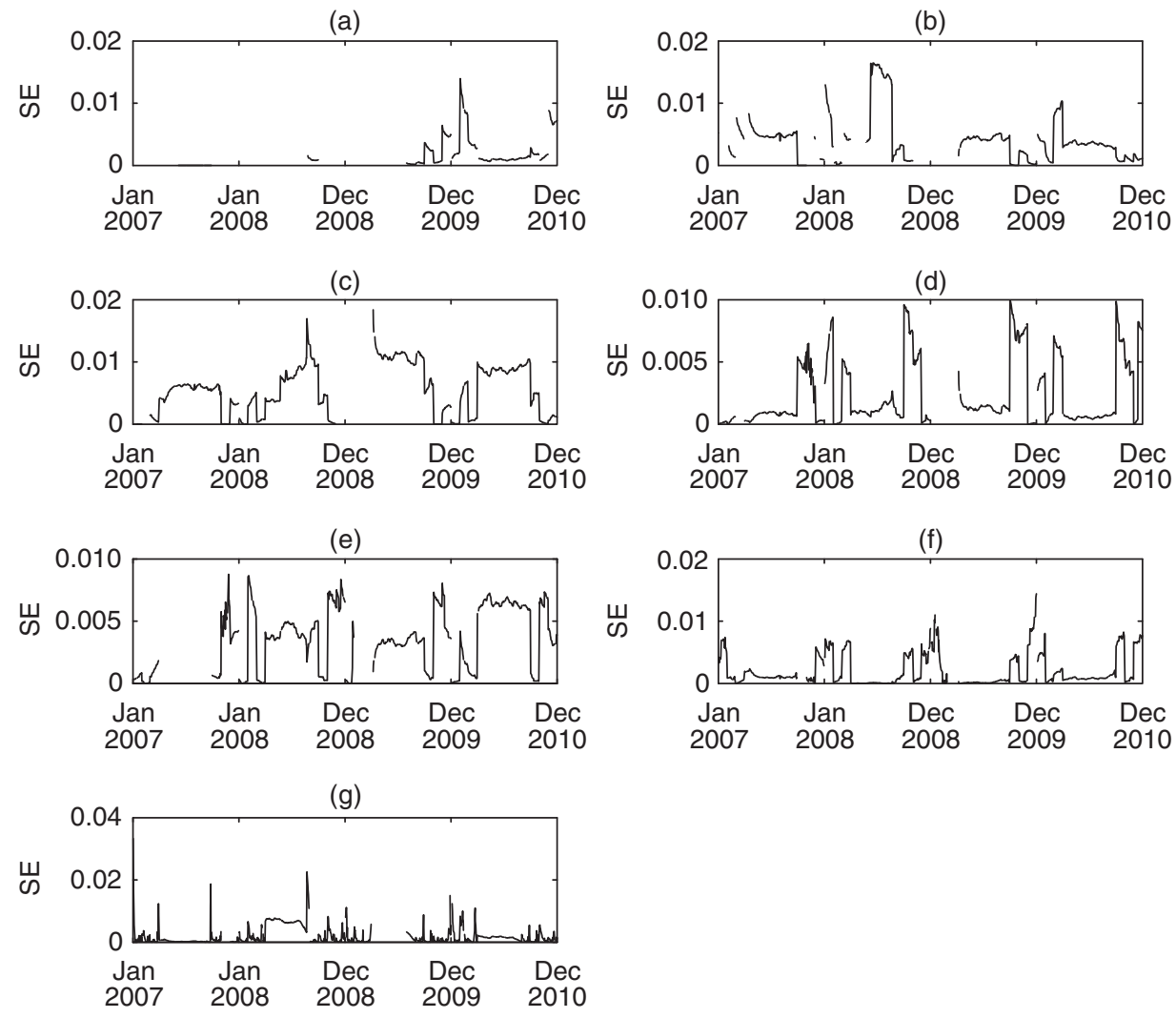

(a) Seventh closest. (b) Sixth closest. (c) Fifth closest. (d) Fourth closest. (e) Third closest. (f) Second closest. (g) Closest. The pricing errors jump when the contracts roll.

In order to calculate the four different expectation terms, we will use the same trick as Zhang (1995), namely rewriting the pdf of the bivariate normal distribution in terms of the marginal pdf of the first variable times the conditional pdf of the second variable given the first variable. Remember that we assume $F_{T}^{E}$ and $F_{T}^{I}$ to be lognormally distributed under $\mathbb{Q}$ (ie, $(X, Y)$ are bivariate normal):

$$
\begin{gathered}
F_{T}^{E}=F_{t}^{E} \mathrm{e}^{\mu_{E}+X}, \\
F_{T}^{I}=F_{t}^{I} \mathrm{e}^{\mu_{I}+Y},
\end{gathered}
$$


FIGURE 11 The time series of squared errors of the percentage differences between fitted and actual natural gas futures prices, when modeled jointly with Chicago HDD futures.
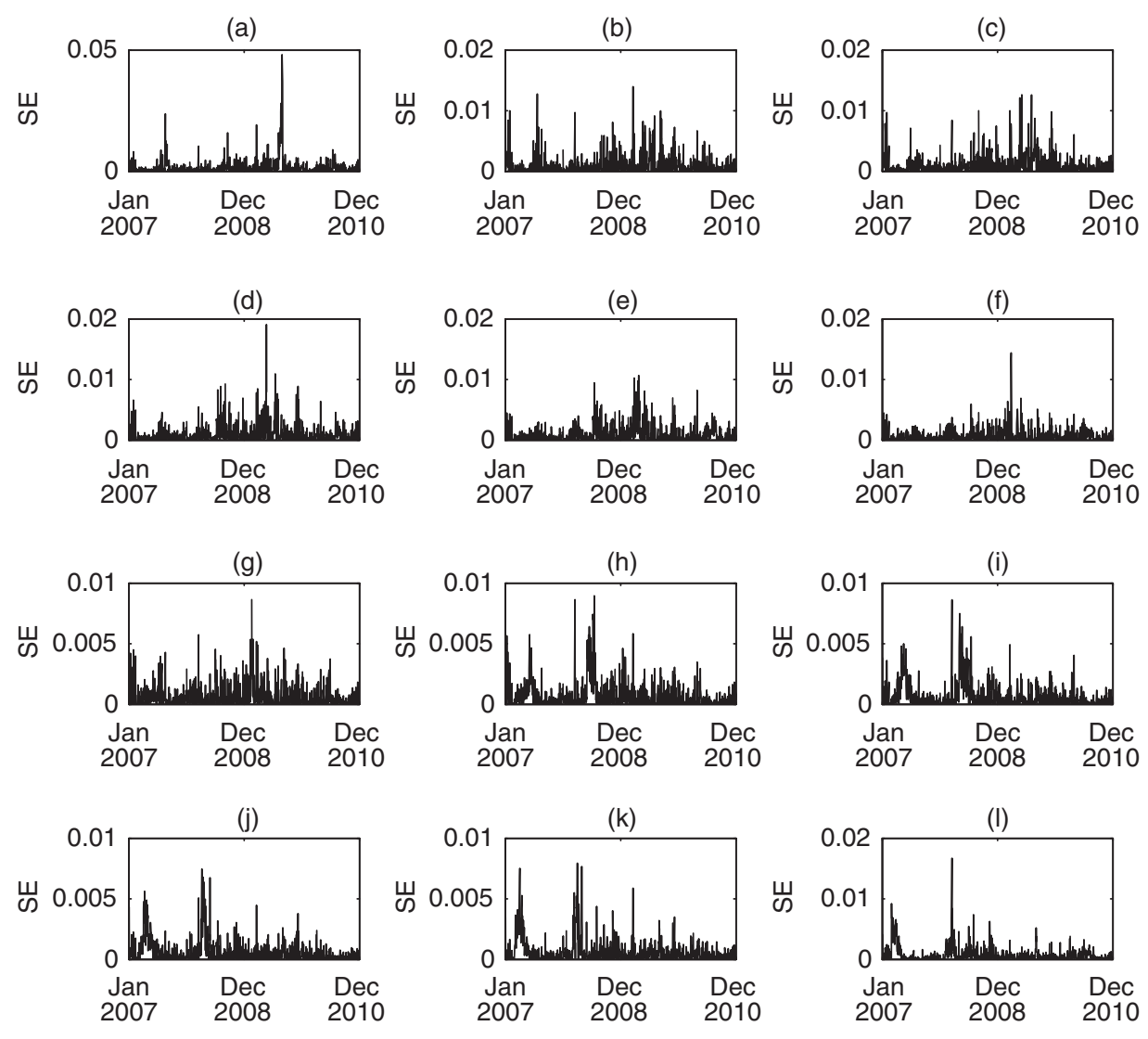

(a) Closest. (b) Second closest. (c) Thirrd closest. (d) Fourth closest. (e) Fifth closest. (f) Sixth closest. (g) Seventh closest. (h) Eighth closest. (i) Ninth closest. (j) Tenth closest. (k) Eleventh closest. (l) Twelfth closest. The pricing errors are largest around the 2008 boom/bust in energy prices.

where $\sigma_{X}^{2}$ denotes the variance of $X, \sigma_{Y}^{2}$ denotes the variance of $Y$, and they are correlated by $\rho_{X, Y}$. Consider the fourth expectation term first:

$$
\begin{aligned}
\mathbb{E}_{t}^{\mathbb{Q}}\left[\bar{K}_{E}\right. & \left.\bar{K}_{I} 1_{\left\{F_{T}^{E}>\bar{K}_{E}\right\}} 1_{\left\{F_{T}^{I}>\bar{K}_{I}\right\}}\right] \\
& =\bar{K}_{E} \bar{K}_{I} \mathbb{E}_{t}^{\mathbb{Q}}\left[1_{\left\{F_{T}^{E}>\bar{K}_{E}\right\}} 1_{\left\{F_{T}^{I}>\bar{K}_{I}\right\}}\right] \\
& =\bar{K}_{E} \bar{K}_{I} \mathbb{Q}_{t}\left(\left\{F_{T}^{E}>\bar{K}_{E}\right\} \cap\left\{F_{T}^{I}>\bar{K}_{I}\right\}\right) \\
& =\bar{K}_{E} \bar{K}_{I} \mathbb{Q}_{t}\left(\left\{F_{t}^{E} \mathrm{e}^{\mu_{E}+X}>\bar{K}_{E}\right\} \cap\left\{F_{t}^{I} \mathrm{e}^{\mu_{I}+Y}>\bar{K}_{I}\right\}\right)
\end{aligned}
$$


TABLE 3 Parameter estimates for the two-dimensional two-factor model with seasonality, when Chicago HDD futures and NYMEX gas futures are modeled jointly.

\begin{tabular}{|c|c|c|}
\hline & Chicago & Gas \\
\hline$\mu$ & $\begin{array}{c}0.0126 \\
(0.0191)\end{array}$ & $\begin{array}{c}-0.0817 \\
(0.0998)\end{array}$ \\
\hline$\kappa$ & $\begin{array}{c}18.8812 \\
(1.3977)\end{array}$ & $\begin{array}{c}0.6034 \\
(0.0317)\end{array}$ \\
\hline$\sigma$ & $\begin{array}{c}0.0379 \\
(0.0051)\end{array}$ & $\begin{array}{c}0.2402 \\
(0.0209)\end{array}$ \\
\hline$v$ & $\begin{array}{c}4.3980 \\
(0.8908)\end{array}$ & $\begin{array}{c}0.6647 \\
(0.0335)\end{array}$ \\
\hline$\rho$ & $\begin{array}{c}-0.5509 \\
(0.0948)\end{array}$ & $\begin{array}{r}-0.7038 \\
(0.0611)\end{array}$ \\
\hline$\alpha$ & $\begin{array}{c}0.0107 \\
(0.0040)\end{array}$ & $\begin{array}{c}-0.3403 \\
(0.0249)\end{array}$ \\
\hline$\lambda$ & $\begin{array}{r}-5.8799 \\
(2.9083)\end{array}$ & $\begin{array}{r}-0.9438 \\
(0.1988)\end{array}$ \\
\hline$\sigma_{\epsilon}$ & $\begin{array}{c}0.0554 \\
(0.0005)\end{array}$ & $\begin{array}{c}0.0199 \\
(0.0001)\end{array}$ \\
\hline$\gamma_{1}$ & $\begin{array}{c}0.8705 \\
(0.0019)\end{array}$ & $\begin{array}{c}0.0499 \\
(0.0003)\end{array}$ \\
\hline$\gamma_{1}^{*}$ & $\begin{array}{c}0.6391 \\
(0.0015)\end{array}$ & $\begin{array}{c}0.0406 \\
(0.0003)\end{array}$ \\
\hline$\gamma_{2}$ & $\mathrm{~N} / \mathrm{A}$ & $\begin{array}{c}0.0128 \\
(0.0003)\end{array}$ \\
\hline$\gamma_{2}^{*}$ & $\mathrm{~N} / \mathrm{A}$ & $\begin{array}{c}0.0270 \\
(0.0003)\end{array}$ \\
\hline$\rho^{W}$ & \multicolumn{2}{|c|}{$\begin{array}{r}-0.2707 \\
(0.0909)\end{array}$} \\
\hline$\rho^{B}$ & \multicolumn{2}{|c|}{$\begin{array}{c}0.1982 \\
(0.0643)\end{array}$} \\
\hline$\ell$ & \multicolumn{2}{|c|}{37023} \\
\hline
\end{tabular}

$$
\begin{aligned}
= & \bar{K}_{E} \bar{K}_{I} \mathbb{Q}_{t} \\
& \times\left(\left\{X>\log \left(\frac{\bar{K}_{E}}{F_{t}^{E}}\right)-\mu_{E}\right\} \cap\left\{Y>\log \left(\frac{\bar{K}_{I}}{F_{t}^{I}}\right)-\mu_{I}\right\}\right) \\
= & \bar{K}_{E} \bar{K}_{I} \mathbb{Q}_{t} \\
& \times\left(\left\{-X<\log \left(\frac{F_{t}^{E}}{\bar{K}_{E}}\right)+\mu_{E}\right\} \cap\left\{-Y<\log \left(\frac{F_{t}^{I}}{\bar{K}_{I}}\right)+\mu_{I}\right\}\right) \\
= & \bar{K}_{E} \bar{K}_{I} M\left(y_{1}, y_{2} ; \rho_{X, Y}\right),
\end{aligned}
$$


where $\left(\epsilon_{1}, \epsilon_{2}\right)$ are standard bivariate normal with correlation $\rho_{X, Y}$ and

$$
\begin{aligned}
& y_{1}=\frac{\log \left(F_{t}^{E} / \bar{K}_{E}\right)+\mu_{E}}{\sigma_{X}}, \\
& y_{2}=\frac{\log \left(F_{t}^{I} / \bar{K}_{I}\right)+\mu_{I}}{\sigma_{Y}} .
\end{aligned}
$$

Next, consider the third expectation term,

$$
\begin{aligned}
\mathbb{E}_{t}^{\mathbb{Q}}\left[F_{T}^{I} \bar{K}_{E} 1_{\left\{F_{T}^{E}>\bar{K}_{E}\right\}} 1_{\left\{F_{T}^{I}>\bar{K}_{I}\right\}}\right] \\
=F_{t}^{I} \bar{K}_{E} \mathrm{e}^{\mu_{I}} \mathbb{E}\left[\mathrm{e}^{Y} 1_{\left\{F_{T}^{E}>\bar{K}_{E}\right\}} 1_{\left\{F_{T}^{I}>\bar{K}_{I}\right\}}\right] \\
=F_{t}^{I} \bar{K}_{E} \mathrm{e}^{\mu_{I}} \mathbb{E}\left[\mathrm{e}^{\sigma_{Y} \epsilon_{2}} 1_{\left\{\epsilon_{1}<y_{1}\right\}} 1_{\left\{\epsilon_{2}<y_{2}\right\}}\right] \\
=F_{t}^{I} \bar{K}_{E} \mathrm{e}^{\mu_{I}} \int_{-\infty}^{y_{2}} \int_{-\infty}^{y_{1}} \mathrm{e}^{\sigma_{Y} \epsilon_{2}} f\left(\epsilon_{1}, \epsilon_{2}\right) \mathrm{d} \epsilon_{1} \mathrm{~d} \epsilon_{2} \\
=F_{t}^{I} \bar{K}_{E} \mathrm{e}^{\mu_{I}} \int_{-\infty}^{y_{2}} \int_{-\infty}^{y_{1}} \mathrm{e}^{\sigma_{Y} \epsilon_{2}} f\left(\epsilon_{2}\right) f\left(\epsilon_{1} \mid \epsilon_{2}\right) \mathrm{d} \epsilon_{1} \mathrm{~d} \epsilon_{2} \\
=F_{t}^{I} \bar{K}_{E} \mathrm{e}^{\mu_{I}} \int_{-\infty}^{y_{2}} \int_{-\infty}^{y_{1}} \mathrm{e}^{\sigma_{Y} \epsilon_{2}} \frac{1}{\sqrt{2 \pi}} \exp \left(-\frac{1}{2} \epsilon_{2}^{2}\right) \\
\quad \times \frac{1}{\sqrt{2 \pi} \sqrt{1-\rho_{X, Y}^{2}}} \exp \left[\frac{-1}{2\left(1-\rho_{X, Y}^{2}\right)}\left(\epsilon_{1}-\rho_{X, Y} \epsilon_{2}\right)^{2}\right] \mathrm{d} \epsilon_{1} \mathrm{~d} \epsilon_{2} .
\end{aligned}
$$

Using the substitution

$$
w=-\epsilon_{1}+\rho_{\epsilon_{1}, \epsilon_{2}} \sigma_{Y} \quad \text { and } \quad z=-\epsilon_{2}+\sigma_{Y},
$$

the exponent in the above expression becomes:

$$
\begin{aligned}
\sigma_{Y} \epsilon_{2}- & \frac{1}{2} \epsilon_{2}^{2}-\frac{1}{2\left(1-\rho_{X, Y}^{2}\right)}\left(\epsilon_{1}^{2}+\rho_{X, Y}^{2} \epsilon_{2}^{2}-2 \rho_{X, Y} \epsilon_{1} \epsilon_{2}\right) \\
=- & \frac{1}{2\left(1-\rho_{X, Y}^{2}\right)} \\
& \times\left(-2 \sigma_{Y}\left(1-\rho_{X, Y}^{2}\right) \epsilon_{2}+\left(1-\rho_{X, Y}^{2}\right) \epsilon_{2}^{2}+\epsilon_{1}^{2}+\rho_{X, Y}^{2} \epsilon_{2}^{2}-2 \rho_{X, Y} \epsilon_{1} \epsilon_{2}\right) \\
= & -\frac{1}{2\left(1-\rho_{X, Y}^{2}\right)}\left(\epsilon_{1}^{2}-2 \sigma_{Y}\left(1-\rho_{X, Y}^{2}\right) \epsilon_{2}+\epsilon_{2}^{2}-2 \rho_{X, Y} \epsilon_{1} \epsilon_{2}\right) \\
= & -\frac{1}{2\left(1-\rho_{X, Y}^{2}\right)}\left(w^{2}+z^{2}-2 \rho_{X, Y} z w-\left(1-\rho_{X, Y}^{2}\right) \sigma_{Y}^{2}\right) \\
= & -\frac{1}{2\left(1-\rho_{X, Y}^{2}\right)}\left(w^{2}+z^{2}-2 \rho_{X, Y} z w\right)+\frac{1}{2} \sigma_{Y}^{2},
\end{aligned}
$$


which enables us to rewrite (A.4) as

$$
\begin{aligned}
& \mathbb{E}_{t}^{\mathbb{Q}}\left[F_{T}^{I} \bar{K}_{E} 1_{\left\{F_{T}^{E}>\bar{K}_{E}\right\}} 1_{\left\{F_{T}^{I}>\bar{K}_{I}\right\}}\right] \\
& =F_{t}^{I} \bar{K}_{E} \mathrm{e}^{\mu_{I}+\left(\sigma_{Y}^{2} / 2\right)} \\
& \quad \times \int_{-\infty}^{y_{2}^{*}} \int_{-\infty}^{y_{1}^{*}} \frac{1}{2 \pi \sqrt{1-\rho_{X, Y}^{2}}} \\
& \quad \times \exp \left[-\frac{1}{2\left(1-\rho_{X, Y}^{2}\right)}\left(w^{2}+z^{2}-2 \rho_{X, Y} z w\right)\right] \mathrm{d} w \mathrm{~d} z \\
& =F_{t}^{I} \bar{K}_{E} \mathrm{e}^{\mu_{I}+\left(\sigma_{Y}^{2} / 2\right)} M\left(y_{1}^{*}, y_{2}^{*} ; \rho_{X, Y}\right),
\end{aligned}
$$

where

$$
y_{1}^{*}=y_{1}+\rho_{X, Y} \sigma_{Y}, \quad y_{2}^{*}=y_{2}+\sigma_{Y} .
$$

The second expectation term can be calculated in the same way as we calculated the third term. The only difference is that we now use the substitution $\bar{w}=-\epsilon_{1}+\sigma_{X}$ and $\bar{z}=-\epsilon_{2}+\rho_{X, Y} \sigma_{X}$ so we can write

$$
\begin{aligned}
& \mathbb{E}_{t}^{\mathbb{Q}}\left[F_{T}^{E} \bar{K}_{I} 1_{\left\{F_{T}^{E}>\bar{K}_{E}\right\}} 1_{\left\{F_{T}^{I}>\bar{K}_{I}\right\}}\right] \\
& =F_{t}^{E} \bar{K}_{I} \mathrm{e}^{\mu_{E}+\left(\sigma_{X}^{2} / 2\right)} \\
& \quad \times \int_{-\infty}^{y_{2}^{* *}} \int_{-\infty}^{y_{1}^{* *}} \frac{1}{2 \pi \sqrt{1-\rho_{X, Y}^{2}}} \\
& \quad \times \exp \left[-\frac{1}{2\left(1-\rho_{X, Y}^{2}\right)}\left(w^{2}+z^{2}-2 \rho_{X, Y} z w\right)\right] \mathrm{d} w \mathrm{~d} z \\
& =F_{t}^{E} \bar{K}_{I} \mathrm{e}^{\mu_{E}+\left(\sigma_{X}^{2} / 2\right)} M\left(y_{1}^{* *}, y_{2}^{* *} ; \rho_{X, Y}\right),
\end{aligned}
$$

where

$$
y_{1}^{* *}=y_{1}+\sigma_{X} \quad y_{2}^{* *}=y_{2}+\rho_{X, Y} \sigma_{X} .
$$

Finally, consider the first expectation term in (A.1),

$$
\begin{aligned}
\mathbb{E}_{t}^{\mathbb{Q}}\left[F_{T}^{E}\right. & \left.F_{T}^{I} 1_{\left\{F_{T}^{E}>\bar{K}_{E}\right\}} 1_{\left\{F_{T}^{I}>\bar{K}_{I}\right\}}\right] \\
& =F_{t}^{E} F_{t}^{I} \mathrm{e}^{\mu_{E}+\mu_{I}} \mathbb{E}_{t}^{\mathbb{Q}}\left[\mathrm{e}^{X+Y} 1_{\left\{F_{T}^{E}>\bar{K}_{E}\right\}} 1_{\left\{F_{T}^{I}>\bar{K}_{I}\right\}}\right] \\
& =F_{t}^{E} F_{t}^{I} \mathrm{e}^{\mu_{E}+\mu_{I}} \mathbb{E}_{t}^{\mathbb{Q}}\left[\exp \left(\sigma_{X} \epsilon_{1}+\sigma_{Y} \epsilon_{2}\right) 1_{\left\{\epsilon_{1}<y_{1}\right\}} 1_{\left\{\epsilon_{2}<y_{2}\right\}}\right] \\
& =F_{t}^{E} F_{t}^{I} \mathrm{e}^{\mu_{E}+\mu_{I}} \int_{-\infty}^{y_{1}} \int_{-\infty}^{y_{2}} \exp \left(\sigma_{X} \epsilon_{1}+\sigma_{Y} \epsilon_{2}\right) f\left(\epsilon_{1}, \epsilon_{2}\right) \mathrm{d} \epsilon_{2} \mathrm{~d} \epsilon_{1} .
\end{aligned}
$$


Using the same trick as before with the substitution $u=-\epsilon_{1}+\rho_{X, Y} \sigma_{Y}+\sigma_{X}$ and $v=-\epsilon_{2}+\rho_{X, Y} \sigma_{X}+\sigma_{Y}$, (A.5) can be written

$$
\begin{aligned}
\mathbb{E}_{t}^{\mathbb{Q}}\left[F_{T}^{E} F_{T}^{I} 1_{\left\{F_{T}^{E}>\bar{K}_{E}\right\}} 1_{\left\{F_{T}^{I}>\bar{K}_{I}\right\}}\right] \\
\quad=F_{t}^{E} F_{t}^{I} \exp \left(\mu_{E}+\mu_{I}+\frac{1}{2}\left(\sigma_{X}^{2}+\sigma_{Y}^{2}+2 \rho_{X, Y} \sigma_{X} \sigma_{Y}\right)\right) M\left(y_{1}^{* * *}, y_{2}^{* * *} ; \rho_{X, Y}\right),
\end{aligned}
$$

where

$$
y_{1}^{* * *}=y_{1}+\rho_{X, Y} \sigma_{Y}+\sigma_{X}, \quad y_{2}^{* * *}=y_{2}+\rho_{X, Y} \sigma_{X}+\sigma_{Y} .
$$

Thus, the expectation of the payoff function is

$$
\begin{aligned}
& \mathbb{E}_{t}^{\mathbb{Q}}\left[\hat{p}\left(F_{T}^{E}, F_{T}^{I}, \bar{K}_{I}, \bar{K}_{E}\right)\right] \\
& \quad=F_{t}^{E} F_{t}^{I} \exp \left(\mu_{E}+\mu_{I}+\frac{1}{2}\left(\sigma_{X}^{2}+\sigma_{Y}^{2}+2 \rho_{X, Y} \sigma_{X} \sigma_{Y}\right)\right) M\left(y_{1}^{* * *}, y_{2}^{* * *} ; \rho_{X, Y}\right) \\
& \quad-F_{t}^{E} \bar{K}_{I} \mathrm{e}^{\mu_{E}+\left(\sigma_{X}^{2} / 2\right)} M\left(y_{1}^{* *}, y_{2}^{* *} ; \rho_{X, Y}\right) \\
& \quad-F_{t}^{I} \bar{K}_{E} \mathrm{e}^{\mu_{I}+\left(\sigma_{Y}^{2} / 2\right)} M\left(y_{1}^{*}, y_{2}^{*} ; \rho_{X, Y}\right) \\
& \quad+\bar{K}_{E} \bar{K}_{I} M\left(y_{1}, y_{2} ; \rho_{X, Y}\right) .
\end{aligned}
$$

Discounting the expected payoff gives us the price of the option.

APPENDIX B. CLOSED-FORM SOLUTIONS FOR $\sigma$ AND $\rho$ IN THE TWO-DIMENSIONAL SCHWARTZ-SMITH MODEL WITH SEASONALITY

$$
\begin{aligned}
\sigma_{X}^{2}= & \int_{t}^{T}\left(\sigma_{E}^{2}+\left(v_{E} \mathrm{e}^{-\kappa^{E}(\tau-s)}\right)^{2}+2 \rho_{E} \sigma_{E}\left(v_{E} \mathrm{e}^{-\kappa^{E}(\tau-s)}\right)\right) \mathrm{d} s \\
= & \sigma_{E}^{2}(T-t)+v_{E}^{2} \int_{t}^{T} \mathrm{e}^{-2 \kappa^{E}(\tau-s)} \mathrm{d} s+2 \rho_{E} \sigma_{E} v_{E} \int_{t}^{T} \mathrm{e}^{-\kappa^{E}(\tau-s)} \mathrm{d} s \\
= & \sigma_{E}^{2}(T-t)+\frac{v_{E}^{2}}{2 \kappa^{E}} \mathrm{e}^{-2 \kappa^{E} \tau}\left(\mathrm{e}^{2 \kappa^{E} T}-\mathrm{e}^{2 \kappa^{E} t}\right) \\
& +2 \frac{\rho_{E} \sigma_{E} v_{E}}{\kappa^{E}} \mathrm{e}^{-\kappa^{E} \tau}\left(\mathrm{e}^{\kappa^{E} T}-\mathrm{e}^{\kappa^{E} t}\right) ; \\
\operatorname{cov}(X, Y)= & \rho_{W} \int_{t}^{T} \sigma_{E} \sigma_{I} \mathrm{~d} s+\rho_{B} \int_{t}^{T}\left(v_{E} \mathrm{e}^{-\kappa^{E}(\tau-s)}\right)\left(v_{I} \mathrm{e}^{-\kappa^{I}(\tau-s)}\right) \mathrm{d} s \\
= & \rho_{W} \sigma_{E} \sigma_{I}(T-t)+\rho_{B} v_{E} v_{I} \mathrm{e}^{-\left(\kappa^{E}+\kappa_{I}\right) \tau} \int_{t}^{T} \mathrm{e}^{\left(\kappa^{E}+\kappa_{I}\right) s} \mathrm{~d} s \\
= & \rho_{W} \sigma_{E} \sigma_{I}(T-t)+\frac{\rho_{B} v_{E} v_{I}}{\kappa^{E}+\kappa^{I}} \mathrm{e}^{-\left(\kappa^{E}+\kappa_{I}\right) \tau}\left(\mathrm{e}^{\left(\kappa^{E}+\kappa^{I}\right) T}-\mathrm{e}^{\left.\left(\kappa^{E}+\kappa^{I}\right) t\right) ;}\right. \\
\rho_{X, Y}= & \frac{\operatorname{cov}(X, Y)}{\sigma_{X} \sigma_{Y}} .
\end{aligned}
$$


When $T=\tau$, this simplifies to

$$
\begin{aligned}
\sigma_{X} & =\sigma_{E}^{2}(\tau-t)+\frac{v_{E}^{2}}{2 \kappa^{E}}\left(1-\mathrm{e}^{-2 \kappa^{E}(\tau-t)}\right)+2 \frac{\rho_{E} \sigma_{E} v_{E}}{-\kappa^{E}}\left(1-\mathrm{e}^{\kappa^{E}(\tau-t)}\right), \\
\rho_{X, Y} & =\frac{\rho_{W} \sigma_{E} \sigma_{I}(\tau-t)+\left(\rho_{B} v_{E} v_{I} / \kappa^{E}+\kappa^{I}\right)\left(1-\mathrm{e}^{-\left(\kappa^{E}+\kappa_{I}\right)(\tau-t)}\right)}{\sigma_{X} \sigma_{Y}} .
\end{aligned}
$$

\section{APPENDIX C. ONE-DIMENSIONAL OPTION PRICES}

In this section, option prices on one underlying are presented. As for the joint case, assume that the dynamics of a gas futures contract is given by:

$$
F_{T}^{E}\left(\tau_{1}, \tau_{2}\right)=F_{t}^{E}\left(\tau_{1}, \tau_{2}\right) \exp \left(\mu_{E}+X\right)
$$

Consider now a call option written on gas futures only. The price $c_{t}$ of this option is then given by the Black (1976) formula, ie,

$$
c_{t}=\mathrm{e}^{-r(T-t)}\left[F N\left(d_{1}\right)-K N\left(d_{2}\right)\right]
$$

where

$$
d_{1}=\frac{\ln \left(F_{t}^{E} / \bar{K}_{E}\right)-\mu_{E}}{\sigma_{X}}, \quad d_{2}=\frac{\ln \left(F_{t}^{E} / \bar{K}_{E}\right)+\mu_{E}}{\sigma_{X}} .
$$

The same formula also applies to an option written only on temperature futures.

\section{APPENDIX D. ESTIMATION USING KALMAN FILTER TECHNIQUES}

Given a set of observed futures prices, it is possible to estimate the parameters using Kalman filter techniques. Let

$$
Y_{n}=\left(f_{t_{n}}^{I}\left(T_{n}^{1}\right), \ldots, f_{t_{n}}^{I}\left(T_{n}^{M_{n}^{I}}\right), f_{t_{n}}^{E}\left(T_{n}^{1}\right), \ldots, f_{t_{n}}^{E}\left(T_{n}^{M_{n}^{E}}\right)\right)^{\prime}
$$

denote the set of $\log$ futures prices observed at time $t_{n}$ with maturities $T_{n}^{1}, \ldots, T_{n}^{M_{n}^{I}}$ for the temperature contracts and maturities $T_{n}^{1}, \ldots, T_{n}^{M_{n}^{E}}$ for the gas contracts. The measurement equation relates the observations to the unobserved state vector $U_{n}=\left(X_{t_{n}}, Z_{t_{n}}\right)^{\prime}$ by

$$
Y_{n}=\mathrm{d}_{n}+C_{n} U_{n}+\epsilon_{n},
$$

where the $\epsilon$ are measurement errors assumed to be independent and identically distributed (iid) normal with zero mean and covariance matrix $H_{n}$. In the present 
framework, we have

$$
\mathrm{d}_{n}=\left(\begin{array}{c}
\Lambda^{I}\left(T_{n}^{1}\right)+A^{I}\left(T_{n}^{1}-t_{n}\right) \\
\vdots \\
\Lambda^{I}\left(T_{n}^{M_{n}^{I}}\right)+A^{I}\left(T_{n}^{M_{n}^{I}}-t_{n}\right) \\
\Lambda^{E}\left(T_{n}^{1}\right)+A^{E}\left(T_{n}^{1}-t_{n}\right) \\
\vdots \\
\Lambda^{E}\left(T_{n}^{M_{n}^{E}}\right)+A^{E}\left(T_{n}^{M_{n}^{E}}-t_{n}\right)
\end{array}\right), \quad C_{n}=\left(\begin{array}{cc}
1 & \mathrm{e}^{-\kappa^{I}\left(T_{n}^{1}-t_{n}\right)} \\
\vdots & \vdots \\
1 & \mathrm{e}^{-\kappa^{I}\left(T_{n}^{M_{n}^{I}}-t_{n}\right)} \\
1 & \mathrm{e}^{-\kappa^{E}\left(T_{n}^{1}-t_{n}\right)} \\
\vdots & \vdots \\
1 & \mathrm{e}^{-\kappa^{E}\left(T_{n}^{M_{n}^{E}}-t_{n}\right)}
\end{array}\right)
$$

and

$$
H_{n}=\left(\begin{array}{cc}
\sigma_{\epsilon, I}^{2} I_{M_{n}^{I}} & 0 \\
0 & \sigma_{\epsilon, E}^{2} I_{M_{n}^{E}}
\end{array}\right)
$$

The state vector evolves according to

$$
U_{n}=c+T U_{n}+\eta_{n},
$$

where $\eta$ is iid normal with a zero-mean vector and covariance matrix $Q$, and where

$$
\begin{aligned}
& c=\left(\begin{array}{c}
\mu^{I}-\frac{1}{2}\left(\sigma^{I}\right)^{2} \\
0 \\
\mu^{E}-\frac{1}{2}\left(\sigma^{E}\right)^{2} \\
0
\end{array}\right) \Delta_{n+1}, \quad T=\left(\begin{array}{cccc}
1 & 0 & 0 & 0 \\
0 & \mathrm{e}^{-\kappa^{I} \Delta_{n+1}} & 0 & 0 \\
0 & 0 & 1 & 0 \\
0 & 0 & 0 & \mathrm{e}^{-\kappa^{E} \Delta_{n+1}}
\end{array}\right), \\
& Q=\left(\begin{array}{cc}
\left(\sigma^{I}\right)^{2} \Delta_{n+1} & 0 \\
0 & \frac{\left(v^{I}\right)^{2}}{2 \kappa^{I}}\left(1-\mathrm{e}^{-2 \kappa^{I} \Delta_{n+1}}\right) \\
\rho^{S} \sigma^{I} \sigma^{E} \Delta_{n+1} & 0 \\
0 & \frac{\rho^{L} v^{I} v^{E}}{\left(\kappa^{I}+\kappa^{E}\right)}\left(1-\mathrm{e}^{\left.-\left(\kappa^{I}+\kappa^{E}\right) \Delta_{n+1}\right)}\right.
\end{array}\right. \\
& \rho^{S} \sigma^{I} \sigma^{E} \Delta_{n+1} \\
& 0 \\
& \left(\sigma^{E}\right)^{2} \Delta_{n+1} \\
& 0 \\
& \left.\begin{array}{l}
0 \\
\mathrm{e}^{\left.-\left(\kappa^{I}+\kappa^{E}\right) \Delta_{n+1}\right)} \\
\mathrm{e}^{\left.-2 \kappa^{E} \Delta_{n+1}\right)}
\end{array}\right)
\end{aligned}
$$




\section{DECLARATION OF INTEREST}

Financial support from "Managing Weather Risk in Electricity Markets” (MAWREM) RENERGI/216096 funded by the Norwegian Research Council is gratefully acknowledged, as is financial support from the NASDAQ OMX Nordic Foundation.

\section{ACKNOWLEDGEMENTS}

We thank participants from the Wolfgang Pauli Institute's Conference on Energy Finance 2012 in Vienna, the Energy Finance Conference 2012 in Trondheim, the 4th International Ruhr Energy Conference (INREC) 2013 in Essen and the Energy Finance Christmas Workshop 2013 in Oslo for helpful feedback and suggestions. Editorial processing of this paper was undertaken by Ruediger Kiesel.

\section{REFERENCES}

Benth, F. E., and Koekebakker, S. (2008). Stochastic modeling of financial electricity contracts. Energy Economics 30(3), 1116-1157.

Benth, F. E., Benth, J. S., and Koekebakker, S. (2008). Stochastic Modelling of Electricity and Related Markets. Advanced Series on Statistical Science and Applied Probability, Vol. 11. World Scientific.

Black, F. (1976). The pricing of commodity contracts. Journal of Financial Economics 3(12), 167-179.

Caporin, M., Prés, J., and Torró, H. (2012). Model based Monte Carlo pricing of energy and temperature quanto options. Energy Economics 34(5), 1700-1712.

Clewlow, L., and Strickland, C. (2000). Energy Derivatives: Pricing and Risk Management. Lacima Publications.

Considine, G. (2000). Introduction to weather derivatives. Working Paper, CME Group. URL: www.cmegroup.com/trading/weather/files/WEA_intro_to_weather_der.pdf.

Davis, A. (2010). A new direction for weather derivatives. Energy Risk. URL: www.risk.net/ energy-risk/feature/1652654/a-direction-weather-derivatives.

Engle, R. F., Mustafa, C., and Rice, J. (1992). Modelling peak electricity demand. Journal of Forecasting 11(3), 241-251.

Garman, M. B., and Kohlhagen, S. W. (1983). Foreign currency option values. Journal of International Money and Finance 2(3), 231-237.

Jørgensen, P. L. (2007). Traffic light options. Journal of Banking and Finance 31(12), 36983719.

Karatzas, I., and Shreve, S. E. (2000). Brownian Motion and Stochastic Calculus. Springer.

Lucia, J. J., and Schwartz, E. S. (2002). Electricity prices and power derivatives: evidence from the Nordic power exchange. Review of Derivatives Research 5, 5-50.

Miltersen, K. R., and Schwartz, E. S. (1998). Pricing of options on commodity futures with stochastic term structure of convenience yields and interest rates. Journal of Financial and Quantitative Analysis 33(1), 33-59. 
Myers, R. (2008). What every CFO needs to know about weather risk management. Working Paper, Storm Exchange, Inc/CME Group. URL: www.cmegroup.com/trading/weather/ files/WeatherRisk_CEO.pdf.

Pérez-González, F., and Yun, H. (2013). Risk management and firm value: evidence from weather derivatives. Journal of Finance 68(5), 2143-2176.

Schwartz, E. S., and Smith, J. E. (2000). Short-term variations and long-term dynamics in commodity prices: implications for valuation and hedging. Management Science 46, 893-911.

Sørensen, C. (2002). Seasonality in agricultural commodity futures. Journal of Futures Markets 22(5), 393-426.

Timmer, R. P., and Lamb, P. J. (2007). Relations between temperature and residential natural gas consumption in the central and eastern United States. Journal of Applied Meteorology and Climatology 46(11), 1993-2013.

Zhang, P. G. (1995). Correlation digital options. Journal of Financial Engineering 4(1), 75-96. 
